\title{
Laser system design for table-top X-ray light source
}

\author{
Anne-Laure Calendron ${ }^{1,2}$, Joachim Meier ${ }^{1,3}$, Michael Hemmer ${ }^{1}$, Luis E. Zapata ${ }^{1}$, Fabian Reichert ${ }^{1,3}$, \\ Huseyin Cankaya ${ }^{1,2,3}$, Damian N. Schimpf ${ }^{1}$, Yi Hua ${ }^{1,3}$, Guoqing Chang ${ }^{1,2}$, Aram Kalaydzhyan ${ }^{1}$, \\ Arya Fallahi $^{1}$, Nicholas H. Matlis ${ }^{1}$, and Franz X. Kärtner ${ }^{1,2,3,4}$ \\ ${ }^{1}$ Center for Free-Electron Laser Science, Deutsches Elektronen Synchrotron, Notkestrasse 85, 22607 Hamburg, Germany \\ ${ }^{2}$ Centre for Ultrafast Imaging, University of Hamburg, Luruper Chaussee 149, 22761 Hamburg, Germany \\ ${ }^{3}$ Department of Physics, University of Hamburg, Luruper Chaussee 149, 22761 Hamburg, Germany \\ ${ }^{4}$ Department of Electrical Engineering \& Computer Science \& Research Laboratory of Electronics, MIT, Cambridge, MA 02139, USA \\ (Received 11 October 2017; revised 22 December 2017; accepted 15 January 2018)
}

\begin{abstract}
We present possible conceptual designs of a laser system for driving table-top free-electron lasers based on terahertz acceleration. After discussing the achievable performances of laser amplifiers with Yb:YAG at cryogenic and room temperature and $\mathrm{Yb}: \mathrm{YLF}$ at cryogenic temperature, we present amplification modules with available results and concepts of amplifier chains based on these laser media. Their performances are discussed in light of the specifications for the tasks within the table-top light source. Technical and engineering challenges, such as cooling, control, synchronization and diagnostics, are outlined. Three concepts for the laser layout feeding the accelerator are eventually derived and presented.
\end{abstract}

Keywords: concept; free-electron laser; lasers; optics

\section{Introduction}

Several laser facilities for strong-field physics experiments such as generation of attosecond pulses ${ }^{[1]}$, electron acceleration $^{[2]}$, proton acceleration ${ }^{[3,4]}$ or X-ray generation ${ }^{[5]}$, have been or are currently being built around the world. These facilities contribute to numerous advances in science, including physics, chemistry, biology, medicine and material science. Their development constantly faces technological and engineering challenges to reach the desired peak power, pulse energy, average power and lifetime.

The 'table-top' free-electron laser (FEL) powered by terahertz $(\mathrm{THz}$ ) radiation proposed in the project AXSIS (Frontiers in attosecond $\mathrm{X}$-ray science: imaging and spectroscopy) in $2016^{[5]}$ is part of the current intense research on alternative technologies to traditional, radio frequency (RF) based FELs $^{[6-8]}$, which are very powerful but complex, large and expensive structures. These devices are thus few in number, resulting in very high demand and very limited access. The scientific community greatly benefits from alternative light sources with reduced size, cost and infrastructure needs. This demand has instigated extensive research efforts aiming

Correspondence to: A.-L. Calendron, DESY - CFEL, Geb. 99/R. O3101, Germany. Email: anne-laure.calendron@desy.de the realization of 'table-top' light sources ${ }^{[9-15]}$. Nonetheless, all the proposed solutions, either directly or indirectly, take advantage from the recent progress in high-energy optical lasers, which are complex facilities.

Building an X-ray light source or other types of light sources entirely based on optical lasers requires a system capable of producing a multitude of diverse laser beams with various characteristics like center wavelength, spectrum, pulse duration and energy to serve as both drivers and diagnostic tools. In particular, for the AXSIS project, ultra-short ultraviolet (UV) pulses are required for electron generation, multiple beams of high-energy picosecond pulses are required for single-cycle $\mathrm{THz}$ generation and high-energy nanosecond (ns) pulses, with tailorable, tunable spectral and temporal profiles, are required for multi-cycle $\mathrm{THz}$ generation. In addition, the facility should run at high repetition rates with femtosecond level synchronization and stabilization. Such a system is highly complex and requires a combination of different laser technologies, some of which must be developed or customized. The right choice of technologies is crucial to its successful implementation and operation.

In this paper, we will first present the AXSIS project and derive the requirements of the driving laser system. Second, the available laser technologies will be reviewed. The third 


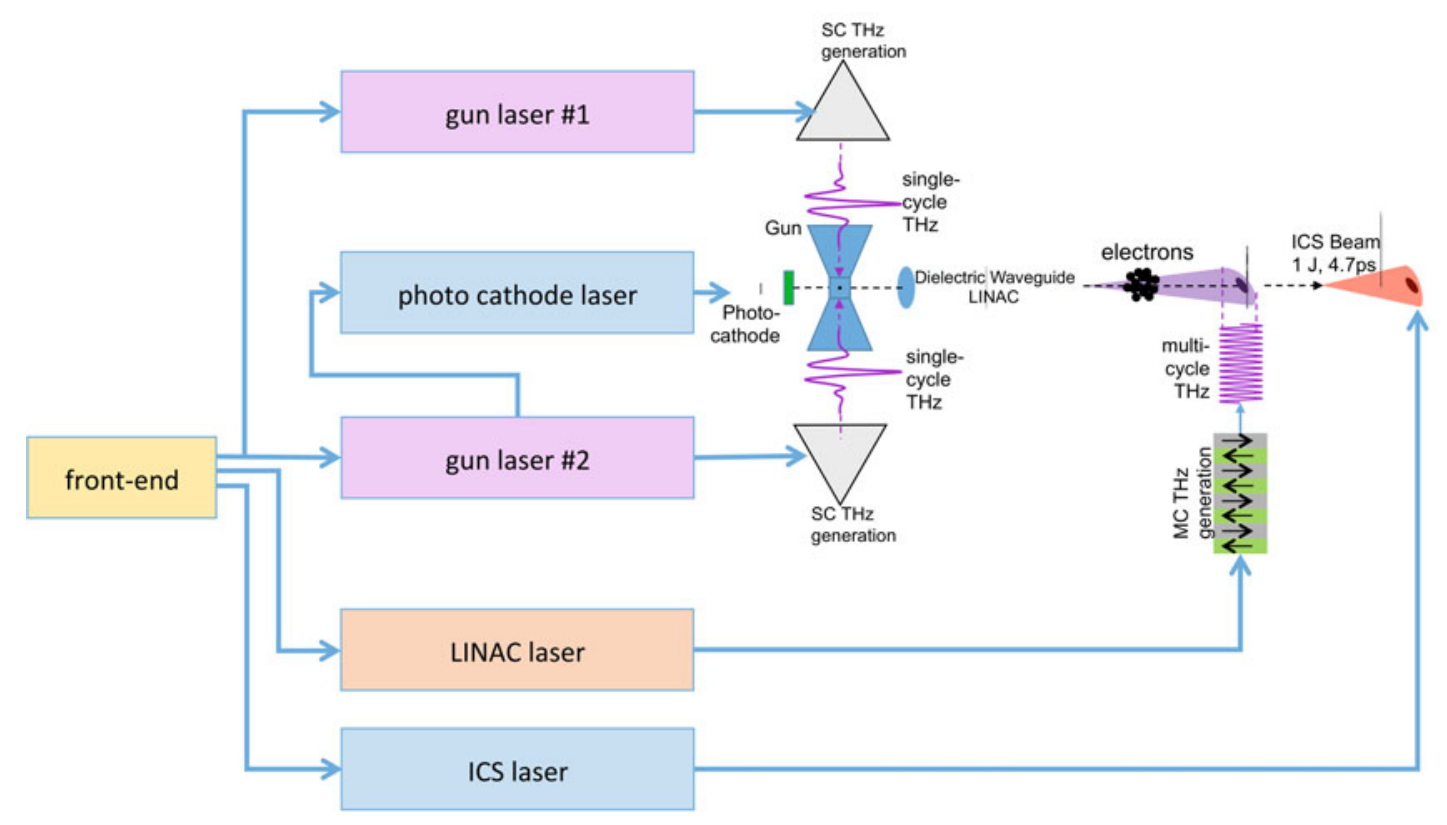

Figure 1. Schematic representation of the THz-driven light source with the driving laser system. SC: single-cycle; MC: multi-cycle, ICS: inverse Compton scattering.

section presents possible modules of the laser system and discusses the first experimental results. Discussion of the laser chains constituted by the described modules tailored for the application within the AXSIS machine is the focus of the fourth section. The main engineering challenges in e.g., synchronization, diagnostics and controls, long-term stability or facility design, will be described and the current status of the implementation will be reported in Section 5. Finally, three possible laser architectures suitable to drive such a table-top light source will be presented and evaluated.

\section{Overall layout of the THz-driven light source}

Figure 1 represents the conceptual layout of the AXSIS machine, including the corresponding high-energy laser drivers. An electron bunch is generated on a photocathode by a low-energy UV pulse. It is initially accelerated to a low relativistic velocity within the gun structure by two counterpropagating, single-cycle $\mathrm{THz}$ waves. Acceleration to higher velocity will be performed by at least one LINAC driven by multi-cycle $\mathrm{THz}$ pulses. An optical undulator (OU) powered by counter-propagating, high-energy infrared (IR) pulses will be used to generate X-rays.

A key technology of this scheme is the use of $\mathrm{THz}$ radiation to accelerate electrons. The high frequency allows acceleration gradients that are 10 to 100 times stronger than in a conventional $\mathrm{RF}$ accelerator ${ }^{[5]}$, resulting in a proportional decrease of the accelerator size and opening the possibility to achieving relativistic electrons within centimeter-scale devices.
To reach kilo electron volt (keV) X-ray photon energies using the OU, electrons with energies in the tens of mega electron volt $(\mathrm{MeV})$ range should be produced, which in turn requires tens of millijoule $(\mathrm{mJ})$ of $\mathrm{THz}$ radiation. Such $\mathrm{THz}$ pulse energies exceed the current state of the art technology by several orders of magnitude. Although various methods ${ }^{[16]}$ exist to generate $\mathrm{THz}$ radiation, laserbased nonlinear optical conversions via difference frequency generation (DFG) or optical rectification (OR) are the only ones suitable for short, high-energy pulses. Both processes allow for $\mathrm{THz}$ generation with intrinsic synchronization and high conversion efficiencies above $1 \%{ }^{[17-22]}$. Thus, laser pulse energies in the range of 0.1 to $1 \mathrm{~J}$ are anticipated for the generation of multi-10-mJ $\mathrm{THz}$ pulses. A primary technological challenge in this approach is the development of laser sources with sufficient pulse energy and optical bandwidth to support the required efficiencies in the DFG or OR process.

The design of the AXSIS gun and LINAC calls for $\mathrm{THz}$ frequencies in the range of $0.1-0.6 \mathrm{THz}$ in order to balance the benefits of high operation frequencies with the drawbacks of small wavelengths ${ }^{[23]}$. These $\mathrm{THz}$ frequencies require optical bandwidths in the range of $1-2 \mathrm{~nm}$ or more at $1 \mu \mathrm{m}$ central wavelength, which can be difficult to achieve at the kilowatt $(\mathrm{kW})$ level.

Also, the OU stage of the scheme presented in Figure $1^{[5]}$ requires a high-energy laser. For example, an electron bunch of $3 \mathrm{pC}$ at $20 \mathrm{MeV}$, upon collision with a $100 \mathrm{~mJ}-1 \mathrm{~J}$, 1 ps optical pulse centered at $1 \mu \mathrm{m}$ wavelength, will produce an X-ray photon beam with a spectrum centered 
Table 1. Summary of the requirements of each laser chain. The THz energy takes into account the transport losses (for single-cycle THz pulses, twice the required energy within the gun is accounted for, and $\sim 1.5$ for multi-cycle $\mathrm{THz}$ pulses).

\begin{tabular}{|c|c|c|c|c|}
\hline & Photocathode laser & Gun lasers & LINAC laser & ICS laser \\
\hline$\overline{T H z}$ energy $[\mathrm{mJ}]$ & & 1 & 30 & \\
\hline IR/UV energy [J] & $100 \times 10^{-9}-200 \times 10^{-9}$ & $0.1-1$ & 1 & $0.1-1$ \\
\hline Conversion efficiency needed & & 2 & 15 & \\
\hline THz pulse structure & & $\mathrm{SC}$ & $\mathrm{MC}$ & \\
\hline THz duration $[\mathrm{ps}]$ & & $<10$ & 200 & \\
\hline IR/UV duration [ps] & $0.04-0.1$ & $0.8-5$ & 200 & $0.8-5$ \\
\hline Central wavelength $[\mathrm{nm}]$ & 253 & $1020-1030$ & & \\
\hline Repetition rate $[\mathrm{Hz}]$ & \multicolumn{4}{|c|}{$100-1000$} \\
\hline IR beam quality & Gaussian & Super-Gaussian & Super-Gaussian & Super-Gaussian \\
\hline Energy stability & & & & \\
\hline Pointing stability & & $<3 \%$ & & \\
\hline
\end{tabular}

at $6 \mathrm{keV}$ and a flux of approximately $10^{6}-10^{7}$ photons per shot ${ }^{[24]}$.

X-ray diffraction investigations of nano-crystals of organic macro-molecules like photosystem $\mathrm{II}^{[25]}$, to be pursued in the AXSIS project, require acquisition and evaluation of a large number of shots, which dictates a high repetition rate for the machine. Based on the current laser technology and possible advances during the time frame of this project, the design of the 1-J laser sources aims at a repetition rate in the range from $100 \mathrm{~Hz}$ up to $1 \mathrm{kHz}$, implying laser average powers in the $\mathrm{kW}$ range.

The AXSIS architecture as in Figure 1 demands five separate laser systems with task-specific properties. Of those five systems, one is tasked to deliver short-pulse, lowenergy UV radiation, three drive $\mathrm{THz}$ generators $(2 \times$ singleand $1 \times$ multi-cycle $\mathrm{THz}$ ) and the last one is used to drive the OU process. The photocathode laser requires only a low pulse energy which can be easily derived from one of the $\mathrm{THz}$ gun lasers. The two systems driving the singlecycle $\mathrm{THz}$ generation are duplicates - one could envisage to split the output of a laser with twice the energy, with the corresponding engineering challenges.

Considering the conversion efficiency from IR to $\mathrm{THz}$ pulses and of the OU process, those laser systems should generate $100 \mathrm{~mJ}$ to $1 \mathrm{~J}$ IR laser pulses. Because of the gain typically reachable within a single laser amplifier, a chain of amplifiers (henceforth the 'laser chain') must be developed. The requirements of each laser chain are summarized in Table 1. The separation of the THz pulses from the IR drivers is critical to avoiding damaging the accelerator, since the laser energy is very high and only percent-level conversion will occur. This will happen by exploiting the different trajectories and indices experienced by $\mathrm{THz}$ and optical waves in the nonlinear process ${ }^{[19,26]}$.

The arrival time of each pulse at its interaction region is critical; consequently, a common oscillator, stabilized in repetition rate, seeds all laser chains to achieve an intrinsically stable synchronization. Furthermore, optical locks between all laser chains are implemented.
The spatial beam profile of the IR lasers is critical for the efficient conversion to $\mathrm{THz}$ radiation, in order to achieve a Gaussian $\mathrm{THz}$ beam (required by the coupling into the gun and LINAC). Similarly, for the OU process, the spatial beam profile is the dominant property affecting the spot size of the high-energy IR beam at the interaction point, which should be as small as few micrometers in diameter. Consequently, the spatial profile required in every laser chain, independent of the concept chosen, should be homogeneous and diffraction limited. Therefore, we utilize amplifiers able to deliver Gaussian and super-Gaussian beam profiles. The required pointing stability is $1 \%$ of the diffraction limit (DL) - the DL expressed in mm-mrad is defined as $\mathrm{DL}=1.22 \lambda / D$, where $\lambda$ is the wavelength and $D$ the diameter of the beam.

For the accelerator, the energy of the $\mathrm{THz}$ pulses has a direct relationship with the final energy of the accelerated electrons and the one of the IR pulses with the energy of the X-ray photons emitted during the industrial control system (ICS) process. The energy stability of the laser chain determines the energy stability of the THz pulses. For both applications, the energy stability of the IR pulses is set to $<0.1 \% \mathrm{rms}$.

\section{Available laser technologies}

High power laser systems rely on a master-oscillator poweramplifier (MOPA) configuration wherein a frontend generates a low power pulse train with the required spectrum and stretching ratio and a power amplifier provides the gain necessary to reach high pulse energy. Since master oscillators which can generate the pulse shapes required for our application are readily available (either commercially or home-built - see Section 4.1), the primary development and engineering challenges are associated with the power amplifiers. 


\subsection{State of the art}

Suitable gain media for amplification of short pulses at high average power are Yb-doped materials, such as Yb:YAG and Yb:YLF ${ }^{[27,28]}$. The small quantum defect of ytterbium minimizes the heat load while the availability of highbrightness pump diodes allows for scaling to high average power ${ }^{[27]}$. Efficient heat removal and mitigation of thermooptic effects have been the key to the continued performance improvements in $\mathrm{Yb}$ diode-pumped solid-state lasers.

Delivering sub-picosecond pulses with energy up to $1 \mathrm{~J}$ at high repetition rates and high average power represents a challenge on the laser source which can be addressed in multiple ways. One solution could be cryogenically cooled ytterbium based laser amplifiers.

Amplification up to $1 \mathrm{~J}$ at $500 \mathrm{~Hz}$ with 5 ps pulse duration has been demonstrated by Reagan et al. ${ }^{[29]}$ using cryogenically cooled Yb:YAG (cryo-Yb:YAG) amplifiers, increased up to $1.5 \mathrm{~J}$ at $500 \mathrm{~Hz}^{[30]}$. Zapata et al. demonstrated up to $160 \mathrm{~mJ}$ at $100 \mathrm{~Hz}^{[31]}$ and $100 \mathrm{~mJ}$ at $250 \mathrm{~Hz}$ using a composite-thin-disk cryo-Yb:YAG amplifier and predict scalability to higher energies ${ }^{[32]}$. Another possibility for high-energy and even shorter pulses is the cryogenically cooled Yb:YLF (cryo-Yb:YLF) laser amplification medium. Amplification has been demonstrated up to $90 \mathrm{~mJ}$ at $100 \mathrm{~Hz}^{[33,34]}$. Simultaneously, room temperature (RT) and commercially available laser sources must be considered. Two particular candidates are: Yb-based lasers with a thin-disk geometry either from Trumpf Scientific $(220 \mathrm{~W}$, $220 \mathrm{~mJ}, 1 \mathrm{kHz}, 1.9 \mathrm{ps}^{[35]}$ ) or from Dausinger and Giesen $\left(120 \mathrm{~W}, 120 \mathrm{~mJ}, 1 \mathrm{kHz},<3 \mathrm{ps}^{[36]}\right)$ and Amphos with the slab geometry 'Innoslab' $\left(1.1 \mathrm{~kW}, 55 \mu \mathrm{J}, 0.6 \mathrm{ps}, 20 \mathrm{MHz}^{[37]}\right.$ and $54 \mathrm{~mJ}, 0.5 \mathrm{~kW}, 1.5 \mathrm{ps}, 10 \mathrm{kHz}^{[38]}$ ).

The Innoslab geometry has demonstrated its advantage for high average powers with limited pulse energies ${ }^{[37]}$. The gain medium is a slab cooled in one direction, perpendicular to the plane of beam propagation; efficient cooling is achieved by keeping this dimension as thin as possible. The beam profile is elliptical due to the minimized crystal height, given by the heat removal along this direction. During propagation through the crystal, the ellipticity increases, and consequently complicates beam shaping and transport after the amplifier. At high energies and short pulses, the B-integral increases and modulation instability starts to appear, limiting the achievable energy. Having said this, a demonstration of $0.5 \mathrm{~J}$ has been made in 2017 with two Innoslabs amplifiers in a row, with Nd:YAG as active medium and $\sim 30 \mathrm{~ns}$ long pulses ${ }^{[39]}-$ at the time of writing this paper, it is questionable if a reduction of the pulse length by a factor of 30 (to maintain a reasonable chirped pulse amplification (CPA) architecture (stretcher/compressor design)) can be achieved.

RT Yb:YAG thin-disk lasers have shown tremendous potential for high average power operation in the continuous wave regime. In this geometry, the heat removal also occurs in the direction perpendicular to the crystal, but the extraction is implemented in the same direction. The beam size scales then with the surface of the gain material, preventing the increase of nonlinear effects. The challenge here resides in the pump absorption through the thin disk and the extraction of the energy. Recent developments toward developing this technology for operation in the $100 \mathrm{~mJ}$ energy range with picosecond duration pulses have been significant enough to qualify these laser systems as potential drivers for our electron acceleration facility. These lasers would either directly power $\mathrm{THz}$ generation setups or be used as seeds for 1-J-class cryogenic amplifier modules. The demonstrated energy, average power and beam profile agree with the range of our required parameters. The narrow spectral bandwidth, limiting the frequency of $\mathrm{THz}$ possibly generated, however might be a bottleneck that necessitates additional investigations to overcome.

Several independent research groups or companies have demonstrated and commercialized RT Yb:YAG-based thindisk amplifiers with operation above $100 \mathrm{~mJ}$ energy and repetition rate up to $5 \mathrm{kHz}$. For instance, Jung et al. reported up to $300 \mathrm{~mJ}$ output energy with a compressed pulse duration as short as 1.8 ps from a thin-disk amplifier ${ }^{[40]}$. Similarly, Nubbemeyer et al. reported a Yb:YAG thin-disk amplifier delivering up to $200 \mathrm{~mJ}$ output energy with a spectrum spanning $1.5 \mathrm{~nm}$ and a measured compressed pulse duration as short as $1.1 \mathrm{ps}$ at $5 \mathrm{kHz}$ repetition rate ${ }^{[41]}$. Independently, Larionov et al. reported a similar architecture amplifier delivering optical pulses with up to $120 \mathrm{~mJ}$ energy at $1 \mathrm{kHz}$ repetition rate, comparable spectral bandwidth and compressed pulse duration ${ }^{[36]}$. With a thick disk of $0.5 \mathrm{~mm}$, energies up to $1.5 \mathrm{~J}$ have been demonstrated at cryogenic temperature $(\mathrm{CT})^{[30]}$.

From the considerations on thermal issues and achievable beam qualities, we decided in the design of the AXSIS laser system to use either the thin-disk geometry laser amplifiers or the traditional rod architecture ${ }^{[27]}$.

\subsection{Discussion and comparison between $Y b: Y A G$ and Yb:YLF lasers}

The electron gun and LINAC may operate with $0.1-0.6 \mathrm{THz}$ frequency radiation ${ }^{[24]}$, which can be obtained via DFG between two near-IR (NIR) frequencies separated by the frequency of the generated $\mathrm{THz}$ wave ${ }^{[42]}$. It corresponds to a separation of $0.3 \mathrm{~nm}$ at $0.1 \mathrm{THz}$ to $1.7 \mathrm{~nm}$ at $0.5 \mathrm{THz}$ for wavelengths close to $1 \mu \mathrm{m}$. As such, the available bandwidth of the laser chain determines the maximum achievable $\mathrm{THz}$ frequency.

Yb:YAG and Yb:YLF laser amplifiers exhibit different spectroscopic, optical and thermal properties (summarized in Table 2), impacting the application (conversion to $\mathrm{THz}$ pulses or ICS) and the scalability of the amplifiers. 
Table 2. Summary of the spectroscopic and thermo-optic properties of Yb:YAG at RT and CT and Yb:YLF at cryogenic temperature.

\begin{tabular}{llll}
\hline Parameter & $\begin{array}{l}\text { Yb:YAG } \\
\text { @ RT }\end{array}$ & $\begin{array}{l}\text { Yb:YAG } \\
\text { @ CT }\end{array}$ & $\begin{array}{l}\text { Yb:YLF } \\
\text { @ CT }\end{array}$ \\
\hline Lifetime $[\mathrm{ms}]$ & 0.95 & 1 \\
Absorption wavelength [nm] & & 940 \\
Emission wavelength [nm] & 1030 & 1029.5 \\
Emission bandwidth $[\mathrm{nm}]$ & $\sim 9$ & $<1.3$ & 938 \\
Absorption cross-section $\left[10^{-20} \mathrm{~cm}^{-2}\right]$ & 0.8 & 1.6 & 1020 \\
Emission cross-section $\left[10^{-20} \mathrm{~cm}^{-2}\right]$ & 2.2 & $\sim 10$ & 10 \\
Saturation fluence $\left[\mathrm{J} \cdot \mathrm{cm}^{-2}\right]$ & 10 & 1.6 & 1.8 \\
Thermal conductivity $\left[\mathrm{W} \cdot \mathrm{m}^{-1} \cdot \mathrm{K}^{-1}\right]$ & 12 & 47 & 20 \\
Nonlinear refractive index coefficient $\left[10^{-16} \mathrm{~cm}^{2} \cdot \mathrm{W}^{-1}\right]$ & 6.2 & 6.2 & 24 \\
$\mathrm{~d} n / \mathrm{d} T\left[10^{-6} \mathrm{~K}^{-1}\right]$ & 7.8 & 0.9 & 1.7 \\
\hline
\end{tabular}

RT-Yb:YAG is a 3-level system with considerable thermal population of the ground state. In addition it shows a small emission cross-section $\left(\sigma_{\mathrm{em}}=2.2 \times 10^{-20} \mathrm{~cm}^{2}\right)$, which increases to $10 \times 10^{-20} \mathrm{~cm}^{2}$ at cryogenic temperature. The limited emission cross-section of RT-Yb:YAG however translates into a relatively broad emission bandwidth $(\Delta \lambda \sim$ $9 \mathrm{~nm}$ ). For instance, mode-locked Yb:YAG thin-disk oscillators support pulse durations as short as $49 \mathrm{fs}^{[43]}$. At high energy $1.5 \mathrm{~nm}^{[41,42]}$ or $1.1 \mathrm{~nm}^{[43]}$, pulse spectral width has been achieved. The bandwidth is limited to $<1.3 \mathrm{~nm}$ at cryogenic temperature ${ }^{[29,32,44]}$, and typically $0.4 \mathrm{~nm}$ pulse spectral width is achieved, limiting the central frequency of generated $\mathrm{THz}$ pulses to $100 \mathrm{GHz}^{[21]}$. Yb:YLF however exhibits a larger gain bandwidth than Yb:YAG at room temperature, which remains relatively broad at cryogenic temperature with $10 \mathrm{~nm}$. After amplification, the spectral width of the pulses reaches $2.1 \mathrm{~nm}$, allowing the generation of $500 \mathrm{GHz}$ radiation.

Yb:YLF and Yb:YAG amplifiers differ also in their scalability to high energies and high average powers. Average powers of $1 \mathrm{~kW}$ are feasible and have been demonstrated with Yb:YAG at room temperature ${ }^{[37,45]}$. A significant advantage is realized when $\mathrm{Yb}$ :YAG is operated at liquid nitrogen temperature ${ }^{[44]}$, because of the improved thermooptic and thermo-mechanical properties. At cryogenic temperature, the thermal conductivity of Yb:YAG increases to $47 \mathrm{~W} \cdot \mathrm{m}^{-1} \cdot \mathrm{K}^{-1}$, allowing an efficient cooling of the gain medium and minimizing the thermo-optic distortions generated. Additionally, the thermal population of the lower laser level is depleted, resulting in four-level rather than quasi-three-level operation, and the saturation intensity and fluence decrease several-fold allowing for efficient extraction of laser power at intensities well below the damage threshold. RT Yb:YAG thin-disk lasers have demonstrated impressive average power performance parameters attained after applying precision engineering and many years of development, however they are limited by the transverse gain they can hold and thus limited by their energy storage capacity. Therefore amplifier laser systems able to provide hundreds of $\mathrm{mJ}$ and on up to $1 \mathrm{~J}$ output based on this rather
Table 3. Description of the main outputs of the frontend.

\begin{tabular}{ll}
\hline $\begin{array}{l}\text { Frontend } \\
\text { output }\end{array}$ & Description \\
\hline$\# 1$ & $\begin{array}{l}\text { Seed for laser for multi-cycle terahertz generation } \\
\text { (two laser lines) }\end{array}$ \\
$\# 2$ & Ultra-short pulse seed for ICS laser-beam line \\
$\# 3$ & Ultra-short pulse seed for gun lasers \\
$\# 4$ & Ultra-short pulse for diagnostics such as electro- \\
& optical sampling \\
\hline
\end{tabular}

mature technology must avail regenerative architectures that can manage beam stability through a multiplicity of passes through gain media, in the order of $\sim 100$; these architectures include several laser heads and electro-optic switches with the concomitant complexity. In contrast, cryo-Yb:YAG affords several engineering advantages: lower saturation fluence ( $\sim 2 \mathrm{~J} / \mathrm{cm}^{2}$ versus $\sim 12 \mathrm{~J} / \mathrm{cm}^{2}$ at room temperature), higher gain and higher absorption cross sections resulting in simpler diode pumping and multi-pass optical architectures that avoid electro-optic switches, $\sim 5$ times higher thermal conductivity, $\sim 1 / 8$ lower thermal-focusing coefficient $(\mathrm{d} n / \mathrm{d} T)$ and $1 / 3$ the expansion coefficient.

In the AXSIS laser system, we consider laser chains based on (i) cryo-Yb:YAG, (ii) cryo-Yb:YLF, and (iii) RT Yb:YAG. In what follows, we will discuss the characteristics of the modules available for building laser amplification chains, while describing in some detail the performance obtained with the technology demonstrators we have already built and are using.

\section{Description of modules}

\subsection{Frontend}

The main function of the frontend is to provide multiple laser seeds for the principal laser systems. The main outputs of the frontend are summarized in Table 3.

Frontend output \#1 consists of two locked single frequency lasers with center wavelengths in the vicinity of 
$1.03 \mu \mathrm{m}$. The frequency separation of the two lasers is set to the required terahertz frequency, which will be generated by the DFG process in periodically poled lithium niobate $\left(\mathrm{LiNbO}_{3}\right)^{[18]}$. The frequency separation of interest is 200$500 \mathrm{GHz}$. One of the single frequency lasers is broadly tunable, which enables tuning the terahertz frequencies with kilohertz $(\mathrm{kHz})$ precision. The LINAC requires terahertz pulses with multiple cycles. The corresponding pulsed laser signal, with adjustable duration between $300 \mathrm{ps}$ and several ns, is generated from the continuous beat signal of the two laser lines by using optical modulators and further amplification. An electro-optic modulator (EOM) as well as an acousto-optic modulator (AOM) is placed within a fiber-based amplification chain. In this way, we can produce pulses at repetition rates up to $2 \mathrm{MHz}$ with energies of approximately $10 \mathrm{~nJ}$. The EOM is controlled by an arbitrary waveform generator (AWG), which can create a variety of pulse shapes. The duration of square pulses can be as short as $300 \mathrm{ps}$ or as long as $\mu \mathrm{s}$. The fiber amplification chain does not operate in the energy-saturated regime, and thus square pulses will maintain their temporal signature. Following the frontend, there will be a regenerative amplifier reducing the repetition rate down to $100 \mathrm{~Hz}-1 \mathrm{kHz}$, and a cryogenically cooled booster amplifier, both of which operate in saturated condition. The operation in saturation will result in pulse distortions in time, which can be pre-compensated by the combination of AWG and EOM ${ }^{[46]}$.

Frontend outputs \#2-\#4 deliver ultra-short pulsed signals. These signals are generated from the frontend's master oscillator, which is a home-built ultra-short pulse fiber laser. The ytterbium-doped fiber oscillator is mode-locked by nonlinear-polarization rotation (NPR). NPR offers experimentally the highest reliability, largest spectrum and longest operating lifetime compared with other mode-locking technologies. The parameters at the oscillator's output are: energy of $1.5 \mathrm{~nJ}$, repetition rate of $70 \mathrm{MHz}$, spectral bandwidth (at $10 \mathrm{~dB}$ ) of $25 \mathrm{~nm}$, compressed pulse duration of $120 \mathrm{fs}$. The repetition rate of the master oscillator is stabilized to $70 \mathrm{MHz}$, chosen so that the temporal pulse-to-pulse spacing of about $14 \mathrm{~ns}$ is larger than twice the rise time of the Pockels cell of any of the subsequent regenerative amplifiers (which is typically around $5 \mathrm{~ns}$ ). The RF reference clock for repetition-rate stabilization is based on an oven controlled crystal oscillator (OCXO) and can be further disciplined by referencing to a GPS signal.

The energy of the repetition-rate stabilized master oscillator is further amplified in polarization-maintaining, corepumped single mode fiber to $>10 \mathrm{~nJ}$. We employ the technique of pre-chirped managed amplification (PCMA), which is a direct nonlinear fiber-based amplification method ${ }^{[47,48]}$. It allows for pulses with durations shorter than $90 \mathrm{fs}$ and high pulse quality (Strehl ratio $>0.9$ ) without the need for long pulse stretching and compression that is typically needed for CPA. After the PCMA, polarization beam splitters split the watt-level output into multiple outputs. As mentioned in Section 2, YLF or YAG are the optimal candidates as host material with center wavelengths at $1019 \mathrm{~nm}$ and $1030 \mathrm{~nm}$, respectively. Band-pass filters in the optical splitter extract the needed wavelengths from the broadband laser output. In this way, we ensure full flexibility of the laser frontend with respect to the type of host material of the following laser amplifiers.

\subsection{Yb:KYW regenerative amplifier}

The reason for using Yb:KYW in the regenerative amplifier is to provide a larger bandwidth than achievable with Yb:YAG or Yb:YLF at RT or CT during the six decades of amplification. Large bandwidth from this seed source counteracts spectral gain narrowing in the highenergy amplifier stages and gives a sufficiently short pulse to drive the optical parametric amplifiers (OPAs) for the UV generation. The design of the $\mathrm{Yb}: \mathrm{KYW}$ regenerative amplifier is based on the laser demonstrated in Ref. [49]: the cavity is symmetric around two laser crystals sharing the heat load equally and allowing amplification to high energies. The two 2\% Yb:KYW, $3 \mathrm{~mm}$ long crystals are pumped by a fiber-coupled laser diode operating in quasi-continuous wave regime where $120-\mathrm{W}$ pump power is split equally between the two crystals.

The design of the cavity, with the crystal located at one Rayleigh length distance from the focus of the laser beam, allows for insensitivity of the output beam parameters to the thermal lens. Hence, stable operation is enabled over a large range of repetition rates limited only by the Pockels cell driver.

The stretching ratio implemented in the demonstration in Ref. [49] was designed to be adapted for amplification up to the $1 \mathrm{~J}$ level; the minimum stretched pulse duration for operation of the regenerative amplifier is however 300 ps according to B-integral calculations.

The first version of this regenerative amplifier has been implemented with standard opto-mechanics and fine-adjustable mirror holders, and operates trouble-free and alignment-free since four years on a daily basis in our lab. For AXSIS, it is engineered to remove degrees of freedom and minimize the beam height on the breadboard to enhance the mechanical stability and lifetime. In this revised version, a water-cooled $2^{\prime \prime}$-breadboard supports smaller plates on top of which the optics are mounted; the beam height above breadboard is $2^{\prime \prime}$, determined by the height of the Pockels cell holder, and halfinch above the inserted plates. This design mitigates cost and difficulty of implementation, while being very stable and reliable.

\subsection{RT Yb:YAG amplifiers}

As a consequence of the material parameters detailed in Section 3.2, the saturation fluence of RT-Yb:YAG typically 
exceeds the damage fluence in standard CPA systems and the small signal gain is limited. This is particularly true in the case of the thin-disk architecture since the length of the gain medium is limited to a few hundreds of micrometers per pass, therefore limiting the net gain per pass. The combination of limited emission cross-section and short crystal length inspires employing an amplifier architecture that supports numerous passes through the gain medium. Regenerative amplifiers have so far been the architecture of choice since tens of passes are typically required to amplify nanojoule (nJ) level pulses to the $100 \mathrm{~mJ}$ level.

All RT Yb:YAG-based regenerative amplifiers reported todate have been focused on optimizing output energy, average power, beam profile and long-term stability. In our case, we are in addition interested in maximizing the amplified spectral bandwidth to match the $\mathrm{THz}$ radiation generation requirements. The regenerative amplifier architecture in conjunction with the wide emission bandwidth of $\mathrm{Yb}: \mathrm{YAG}$ at room temperature offers opportunities to maximize emission bandwidth via either injection of high-energy broadband pulses or via intra-cavity spectral shaping. Considering the empirical formula established by Rouyer et al. ${ }^{[50]}$ :

$$
\Delta \lambda=\frac{\Delta \lambda_{0}}{\sqrt{1+\ln G\left(\frac{\Delta \lambda_{0}}{\Delta \lambda_{\text {Fluo }}}\right)^{2}}},
$$

where $G$ is the total gain of the amplifier, $\Delta \lambda_{\text {Fluo }}$ is the spectral bandwidth of the gain medium and $\Delta \lambda_{0}$ is the spectral bandwidth of the input pulse, one can compute the amplified emission bandwidth as a function of the seed energy for a pulse derived from a Yb:KYW regenerative amplifier with up to $5 \mathrm{~nm}$ of spectral bandwidth seeded into a Yb:YAG thin-disk regenerative amplifier $\left(\Delta \lambda_{\text {Fluo }}=5 \mathrm{~nm}\right.$, FWHM of available of emission) shown in Figure 2. Intracavity spectral shaping via either thin etalons or narrowband volume Bragg grating is another option, yet it adds complexity to the cavity design and potentially adds losses to an amplifier showing low gain per pass.

Maintaining a wide spectral band within the regenerative amplifier is also beneficial to minimize the accumulation of nonlinear phase typically plaguing thin-disk regenerative amplifiers as the stretched pulse duration increases linearly - in first approximation - with the spectral width of the amplified pulse. Considering a stretching ratio as large as $650 \mathrm{ps} / \mathrm{nm}$ - a parameter we have safely demonstrated in our laboratory and have been operating for several years - and an amplified bandwidth inside the cavity as wide as $2 \mathrm{~nm}$, pulses as temporally stretched as $1.3 \mathrm{~ns}$ could be employed.

The damage fluence of a typical optical coating or material lays in the $F_{\text {ref }}=10 \mathrm{~J} / \mathrm{cm}^{2}$ range at $\tau_{\text {ref }}=10 \mathrm{~ns}$ pulse duration and scales according to pulse duration as

$$
F_{\text {damage }}=F_{\text {ref }} \sqrt{\frac{\tau}{\tau_{\text {ref }}}},
$$

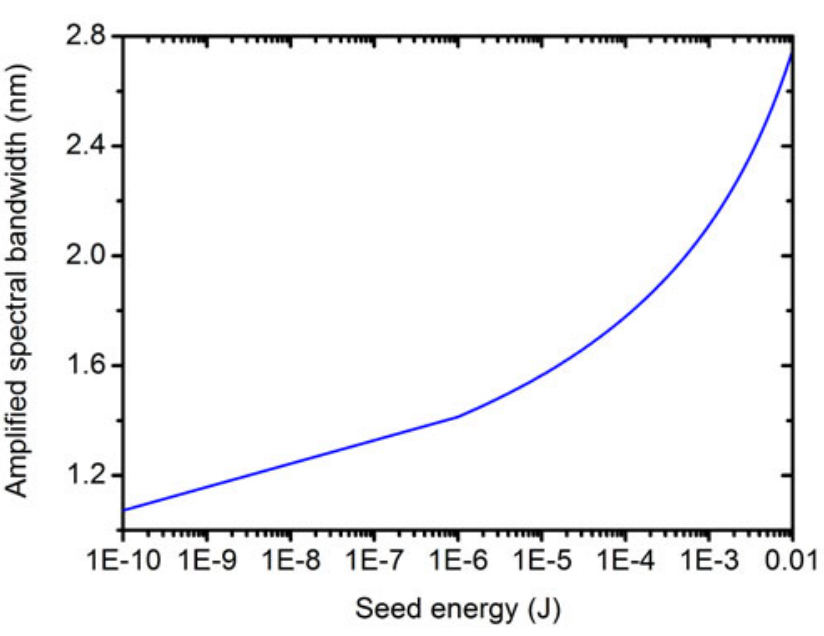

Figure 2. Computed amplified spectral bandwidth as a function of seed energy in a Yb:YAG thin-disk regenerative amplifier $\left(\Delta \lambda_{\text {Fluo }}=5 \mathrm{~nm}\right)$.

results in a maximal tolerable intra-cavity fluence of $3.6 \mathrm{~J} / \mathrm{cm}^{2}$ for $1.3 \mathrm{~ns}$ stretched pulses, still below the saturation energy $F_{\text {Sat }} \sim 9 \mathrm{~J} / \mathrm{cm}^{2}$. Considering a design safety margin of a factor of 2.5 , we set the maximum fluence on critical optics (Pockels cell and gain medium) to $\sim 1.5 \mathrm{~J} / \mathrm{cm}^{2}$ which results in beam sizes as large as $4 \mathrm{~mm}$ diameter at $1 / \mathrm{e}^{2}$ and in an operation of the amplifier away from saturation. Operating a stable cavity with such large beams requires careful cavity design to ensure stable operation in the TEM 00 mode and insensitivity to mechanical disturbances. Similarly, operation of such an amplifier in the unsaturated regime sets stringent requirements on the stability of both the pump and seed lasers. We have however already demonstrated successful operation of a Yb:YLF cryogenic regenerative amplifier operated in a similarly unsaturated regime with output energy stability in the percent range ${ }^{[31]}$.

Fixing the mode size at the gain medium determines much of the energetics behavior of the amplifier as well as the thermal loading of the gain medium. Our current numerical simulations of the energetics are in agreement with the reported results: several tens of passes are required in the gain medium in order to reach the $100 \mathrm{~mJ}$ level. Operation at $100 \mathrm{~Hz}$ repetition rate is preferable to avoid the regime of period doubling observed in regenerative amplifiers operated at a period close to the inverse of the excited lifetime of the dopant ion in the gain medium. It is nonetheless possible to operate at $1 \mathrm{kHz}$ repetition rate, yet care must be taken to operate in the stable regime of period doubling or on the edge of bifurcation.

\section{4. $100 \mathrm{~mJ}$ and $1 \mathrm{~J}$ cryo-Yb:YAG amplifiers}

We are in the process of constructing several engineered prototypes based on various types of the high power thin- 


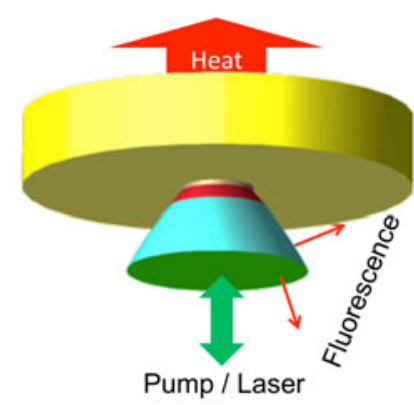

Figure 3. The cryogenic composite thin disk: in our approach, a thin $\mathrm{Yb}$ :YAG gain sheet is diffusion bonded to a thicker index-matched cap on one face while the other face is HR coated and soldered to a backplane highperformance cooler. See text for details.

disk amplifier: a first stage of the composite-thin-disk multipass amplifier ${ }^{[31,32]}$ was first reduced to practice at $100 \mathrm{~mJ}$ per pulse, demonstrated recently. It will serve as the preamplifier for the $1 \mathrm{~J}$ scaled-up amplifier which is under development.

The Yb:YAG $100 \mathrm{~mJ}$ and $1 \mathrm{~J}$ amplifiers are based on a cryogenic composite-thin-disk and multi-pass, relayimaging architecture. The gain-element geometry is resistant to amplified spontaneous emission (ASE), which enables operation at high gain. The strict image relayed multipass architecture allows spatial filtering with every pass to avoid damaging intensity ripples, whereby the laser input efficiently and smoothly saturates the stored energy to deliver high-energy ultrafast pulses with diffraction limited beam quality at high repetition rates.

The enhancements of the composite-thin-disk approach over the traditional approach are detailed in Figure 3. On the cooled face, the laser-grade high-reflector exists in intimate contact - through soldering - with a high performance cooler. The opposite face of the thermally loaded gain sheet is diffusion-bonded to an index-matched 'cap' of undoped YAG. The purpose of the undoped cap is three-fold:

(1) Considerably facilitating the pumping by cryogenic operation: A large amount of diode power can be absorbed in a single 'bounce' avoiding the multi-passing necessary at room temperature, taking advantage of the inherent brightness of modern diode stacks.

(2) Maximizing aperture size: ASE limits the aperture. Compared to the traditional thin disk, the composite thin disk dilutes spontaneous emission into the undoped cap where it is guided toward the edges and ejected. This dramatically reduces ASE loss of gain due to extending the aperture dimensions.

(3) Minimizing deformations: The undoped cap adds strength. Stiffness increases in proportion to the cube of the thickness, minimizing wavefront errors.
In the one-dimensional (1D) thin-disk geometry, the temperature rises uniformly and constantly. The diffusion-bonded undoped cap does not compromise this thermal advantage and does not affect the 1D thermal distribution in the gain sheet.

This composite-thin-disk gain-element geometry is being scaled to store higher energy offering the possibility to reach $1 \mathrm{~J}$ output energy. To extend the results of our $100 \mathrm{~mJ}$ multi-pass amplifier to the 1-J level, the aperture diameter must increase from $4 \mathrm{~mm}$ to $16 \mathrm{~mm}$ while the aspect ratio (diameter to thickness) remains the same in order to scale the ASE rejection. The shape of the edges is also maintained to avoid recirculation and the undoped cap thickness increases proportionately. Calculations show a B-integral value of $\sim 1$ for the multi-pass design.

The $100 \mathrm{~mJ}$ pulses of the first amplifier (shown in Figure 4(a)) will be used as input to the next step, which scales the performance to $1 \mathrm{~J}$ pulses and is currently under construction. A larger but still compact multi-pass laser system design based on similar core technology is completed. The engineering details of this system are illustrated in Figure 4(b). Presently, we are in the final phases of procurement and we estimate activation of the 1-J laser in 2018. The pointing stability after the $1 \mathrm{~J}$ amplifier is expected to be $1 \%$ of the diffraction limit; for a beam with $20 \mathrm{~mm}$ diameter, this corresponds to $0.6 \mu \mathrm{rad}$, which is the minimum physically achievable limit. Taking into account noise sources, e.g., vibrations coupled to mirror holders, we expect $2 \mu$ rad pointing stability. If the $100 \mathrm{~mJ}$ cryo-Yb:YAG amplifier is seeded with $1 \%$ rms energy stability, then the output of the $100 \mathrm{~mJ}$ and $1 \mathrm{~J}$ cryo-Yb:YAG amplifiers will have an energy stability $<2 \%$ rms.

Yb:LuAG emerged recently and is a suitable alternative to Yb:YAG, with higher thermal conductivity at high doping levels and increased emission cross-section but narrower spectral bandwidth $^{[51]}$. We envisage further development using Yb:LuAG.

\subsection{Cryo-YLF laser amplifiers}

After stretching, the cryo-YLF laser chain comprises a regenerative amplifier and a booster amplifier ${ }^{[33,34]}$. The stretcher and compressor are described in Section 4.6.

The regenerative amplifier features a ring-type cavity consisting of four mirrors, a Pockels cell and half-wave plate, two thin-film polarizers (TFPs) and a cryo-Yb:YLF crystal $^{[33,34]}$. The pump power is provided by a fibercoupled $300 \mathrm{~W}$ diode array operated in the pulsed regime. The cavity length of the regenerative amplifier is set to $1.5 \mathrm{~m}$ as a compromise between a small footprint and a large enough spot size. A small footprint enables long enough propagation time to ensure full switching of the Pockels cell high voltage during a round trip in the cavity, whereas a large enough spot size in the gain medium and Pockels 

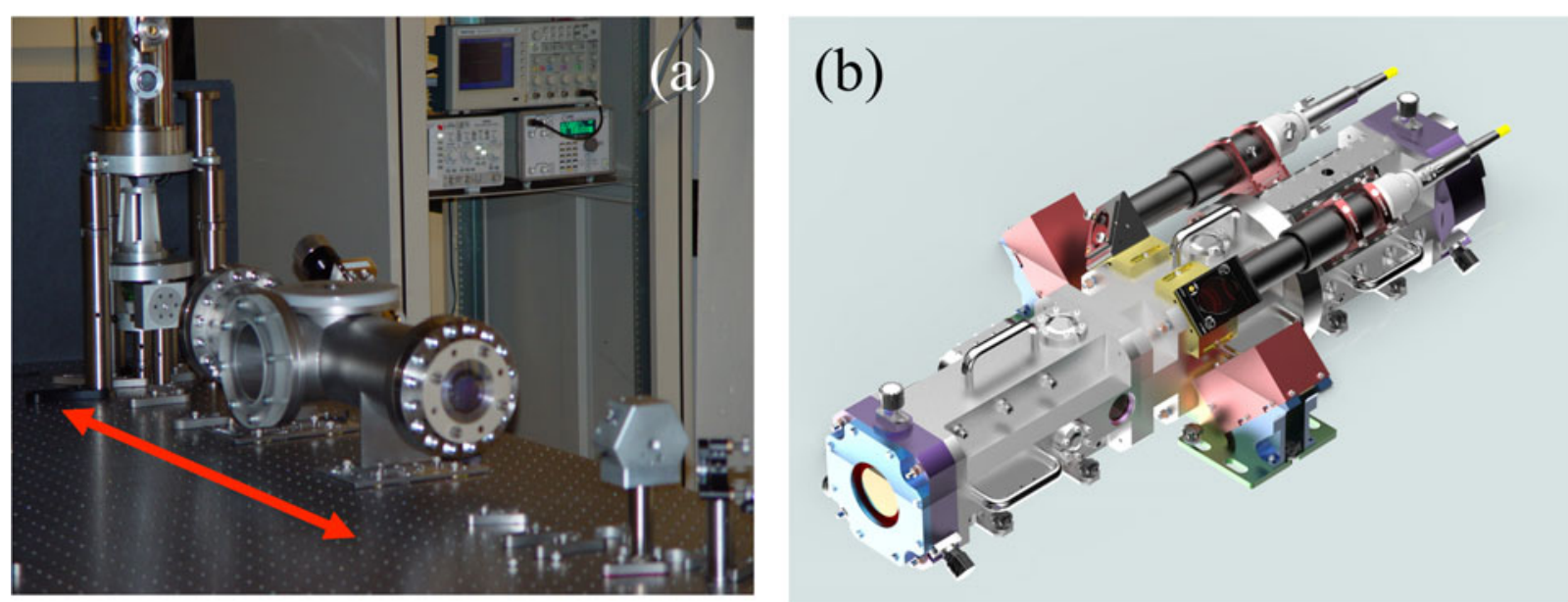

Figure 4. Photographs of the (a) $100 \mathrm{~mJ}$ and (b) $1 \mathrm{~J} \mathrm{Yb}: \mathrm{YAG}$ amplifier.
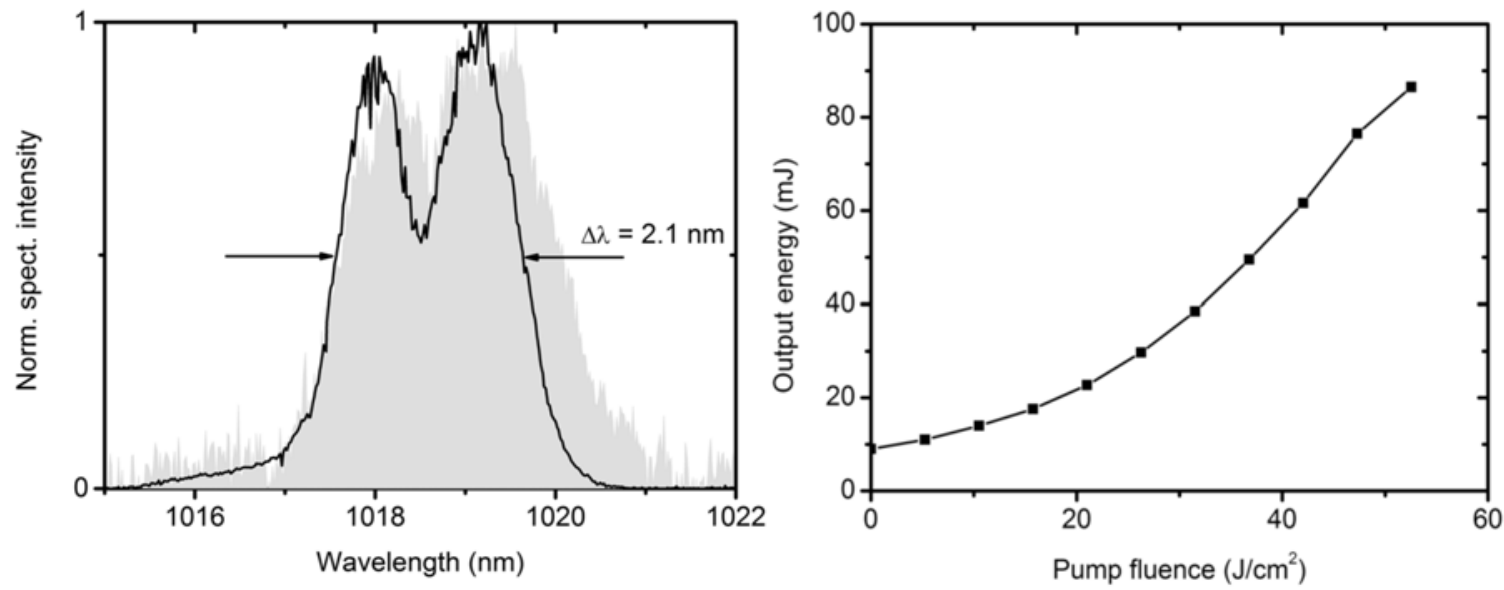

Figure 5. (a) Measured output spectrum (black line) at the $10 \mathrm{~mJ}$ energy level along with seed spectrum (grey shaded region). (b) Measured output energy versus pump input fluence characteristics showing an output energy $\sim 90 \mathrm{~mJ}$ at full pump power.

cell leads to damage free operation at the $10-15 \mathrm{~mJ}$ output energy level. The Pockels cell is chosen to be a dual crystal rubidium titanyle phosphate (RTP) cell with a $6 \mathrm{~mm}$ aperture allowing a low $(\sim 3 \mathrm{kV})$ half-wave voltage of operation and fast switching of the cell. The gain medium is a $5 \mathrm{~mm}$ thick, 25\%-doped Yb:YLF crystal. The regenerative amplifier delivers routinely $10 \mathrm{~mJ}$ output energy at up to $100 \mathrm{~Hz}$ repetition rate in an excellent spatial intensity profile, with an amplified spectral width of $\sim 2.1 \mathrm{~nm}$ and shot-to-shot energy instabilities less than $\pm 0.75 \%$ rms over $1 \mathrm{~h}$.

In order to reach the targeted $100 \mathrm{~mJ}$ output energy, we designed and implemented an additional 4-pass amplifier, which relies on polarization switching and features two $1 \mathrm{~cm} \times 1.5 \mathrm{~cm} \times 2.5 \mathrm{~cm}, 0.5 \%$ doped cryo-Yb:YLF crystals (2.5 cm length) pumped by a $2.2 \mathrm{~kW}$ fiber-coupled diode array operated in pulsed mode. This amplifier is currently delivering up to $90 \mathrm{~mJ}$ energy at $10 \mathrm{~Hz}$ (Figure 5(b)) with an excellent spatial profile and an energy stability following that of the regenerative amplifier. The measured input and output spectra of the regenerative amplifier and the output energy versus pump input fluence of the booster are summarized in Figure 5(a).

\subsection{Stretchers and compressors}

The stretchers and compressors, though having different specifications depending on the amplifying medium, exhibit the same engineering challenges. The $0.3 \mathrm{~nm}$ or $2.1 \mathrm{~nm}$ wide pulses at the output of the Yb:YAG or Yb:YLF amplifiers respectively have to be stretched to $0.8 \mathrm{~ns} / \mathrm{nm}$ and $0.33 \mathrm{~ns} / \mathrm{nm}$ (to $240 \mathrm{ps}$ and $800 \mathrm{ps}$ stretched pulses after amplification) in order to minimize nonlinear effects and reduce the intensity of the pulses well below damage threshold.

Different technologies make such stretching ratios achievable, including the traditional Offner stretcher, chirped fiber and volume Bragg gratings (CFBG and CVBG), which are now commercially available. The strong benefit of CFBG and CVBG over the grating based Offner stretcher is the compactness of the stretcher for a fixed stretching ratio and the possible compensation of higher order dispersion. Due 

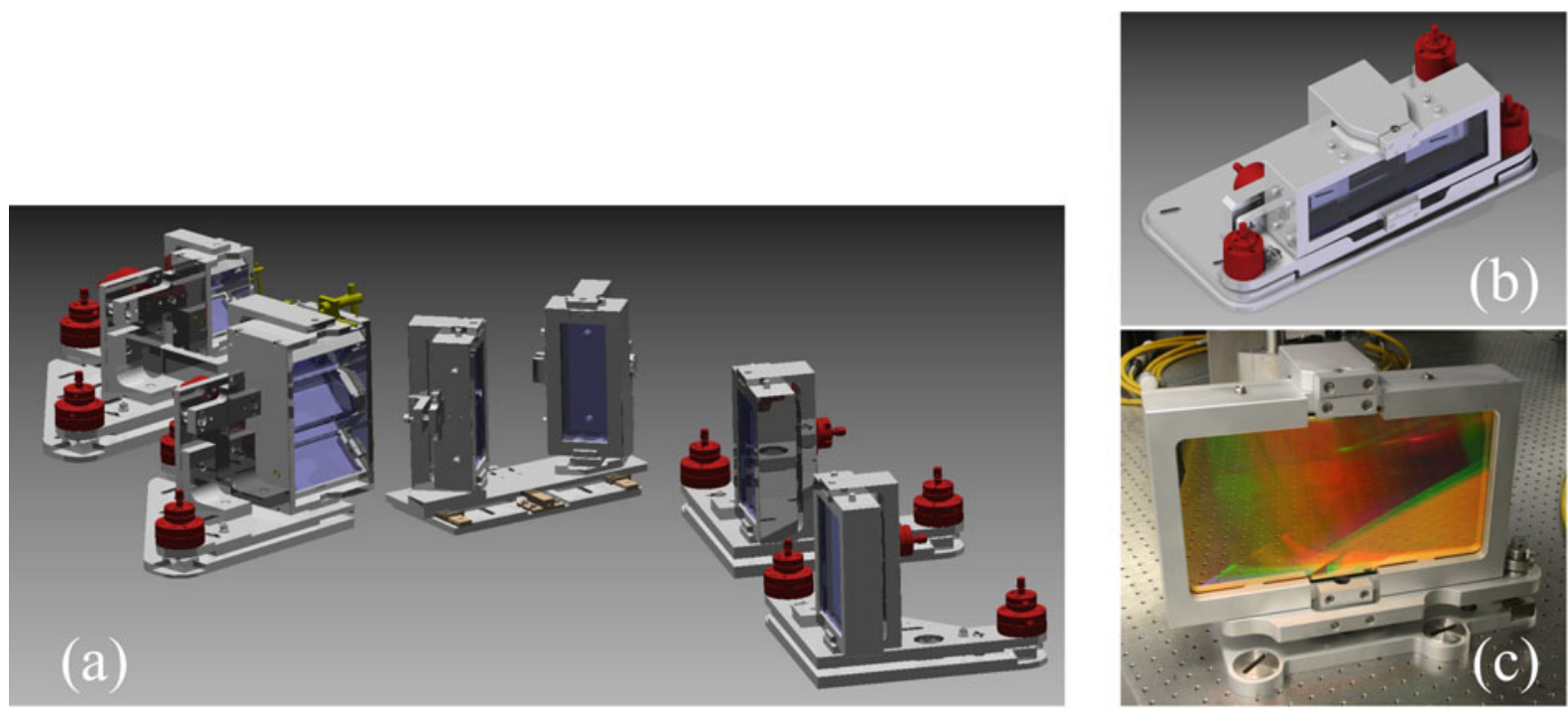

Figure 6. CAD modeling of (a) the grating compressor currently in use after a Yb:YAG high-energy amplifier and (b) the holder of the large grating in the first compressor built in our lab after the Yb:KYW regenerative amplifier ${ }^{[47]}$. (c) A newer version of the grating holder, implemented for the Yb:YLF laser system.

to manufacturing constraints, the CFBGs can be written over a longer length (up to $300 \mathrm{~mm}$ ) compared with CVBGs (up to $75 \mathrm{~mm}$ ). As a result, the net dispersion added to a pulse is higher using CFBGs than CVBGs in a singlepass architecture. The net dispersion can be increased by multi-passing the chirped Bragg grating (CBG), but insertion losses and imperfection of the CBGs limit the amount of possible passes in practice. Temperature tuning on the CFBGs allows for fine-tuning of the dispersion curve, i.e., fine adjustment of high-order dispersion after compression. Our desired stretching ratios and pulse bandwidth demand a cascade of 4-5 CVBGs. Therefore, it is more convenient to implement CFBGs as stretcher for this laser system.

The ultra-short pulse outputs from the splitter of the frontend should be stretched before being used in any subsequent regenerative amplifiers of the ICS or electron gun laser systems. We employ a stretcher module that is completely fiber-based ensuring long-term stability. It consists of two CFBGs. CFBG 1 is followed by an ytterbium-doped fiber amplifier (YDFA) 1 and another CFBG 2 and YDFA 2. To monitor the long-term system performance and to detect degradation of components, a $1 \%$ fiber tap coupler after each amplifier stage is inserted and directly coupled to a photodiode and permanently monitored. All these fiber components are polarization-maintaining (PM) to ensure environmentally independent performance of the stretcher modules. The operation of the CFBG can be adjusted to the center laser wavelength of the subsequent amplifiers, i.e., for $1018 \mathrm{~nm}$ in case of Yb:YLF or for $1030 \mathrm{~nm}$ in case of Yb:YAG. A high isolation isolator is placed behind the output of the stretcher modules. The output energy of approximately $15 \mathrm{~nJ}$ serves as input for the regenerative amplifiers.
On the compressor side though, technological alternatives reduce to the grating based Treacy compressor ${ }^{[52,53]}$, as the energy is too high for the achievable aperture of CFBG or CVBGs. The larger the stretching ratio, the longer the distance between the gratings, i.e., the larger the footprint of the compressor. For the Yb:YAG laser chain, the proposed compressor with 1740 lines $/ \mathrm{mm}, 60^{\circ}$ angle of incidence and $1.09 \mathrm{~m}$ perpendicular distance, is folded between the two gratings, where two passes are implemented (i.e., the beam hits four times each grating) decreasing the footprint to $1 \mathrm{~m} \times 2 \mathrm{~m}$. The high diffraction efficiency $>97.5 \%$ of the multi-layer dielectric gratings from Plymouth Grating Laboratory grants an overall compression efficiency above $80 \%$. Such compressor has been twice implemented in our lab, with special care given to the holders of the large optics, as shown in Figure $6^{[54]}$. The number of degrees of freedom has been reduced between both implementations (compare Figures 6(a) and 6(b)) to increase stability. The angle of incidence of the grating and their parallelism is critical to compress the pulses without introducing spatial chirp. Hence, differential screws should be implemented on the angle alignment. Between the first two versions, noticeable improvement of the pointing stability has been made - in the first version, some realignment has to be made every 2-3 months. The measured drifts on the two grating holders of the second version are measured between $0.8 \mu \mathrm{rad} / \mathrm{h}$ and $19 \mu \mathrm{rad} / \mathrm{h}$ along $x$ and $y$ axes. This holder has been once more iterated for the compressor located after the Yb:YLF laser amplifier, as shown in Figure 6(c).

The prevalent use of large optics in our setup calls for special care to their specifications. The turning mirrors are thick to prevent bending along their length, which would 


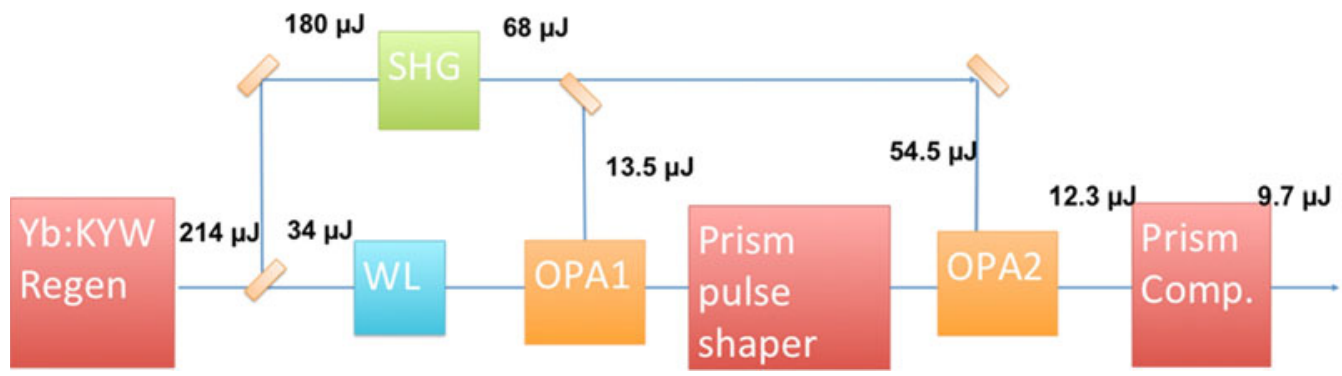

Figure 7. Schematic of the two-stage OPA system to drive the UV generation setup. In the prism compressor located between the two OPA stages, a pulse shaper is implemented: knifes block the highest and lowest spectral components. WL: white-light generation, SHG: second harmonic generation, Comp: compressor.

be detrimental to the beam quality and pulse compression particularly at the regions, where the beam is highly spatially chirped (after the second grating, on the roof mirrors to change the beam height). The surface quality (polishing and scratch/digs) is specified in agreement with the effective size of the beams on the mirrors: for example, on the roof mirrors located after the second grating, where the beam is highly spatially chirped, the flatness is defined as $\lambda / 10$ over the full aperture, whereas on the turning mirrors in between the two gratings, even if the beam is hitting these mirrors four times, a dynamic test area of $50 \mathrm{~mm}$ is sufficient.

During propagation, spatially quasi-flat-top or superGaussian beams develop ripples, which might cause damage on the optics in the compressor. There exist some solutions to prevent this: one is to implement the compressor so that the beam remains over the full distance in the compressor in the near field; for this, one can use a configuration with relay imaging, similar to an Offner stretcher. In the case of a double pass compressor, another solution is to relay image the beam between the two passes when it remains over one pass within the near-field range. With these two solutions, the footprint of the compressor increases a lot, due to the very long focal lengths of the lenses required. A third solution is to let the beam propagate so that the ripples diffract and use the far field of the beam; the inconvenience of this method, that we are currently evaluating, is the loss of $10 \%-20 \%$ of the energy, depending on the exact super-Gaussian order of the beam.

At high average powers, even the residual absorption of the coatings might lead to heating with impact on the alignment or on the wavefront. The multi-layer dielectric gratings we currently use have an efficiency of $97 \%-98 \%$ and the remaining energy is mainly reflected into the 0th order. Thermal effects can also be minimized by using Zerodur substrates. A kW-class compressor has also been demonstrated with dielectric gratings in Ref. [41].

\subsection{UV generation}

In conventional electron guns, electron bunches are generated on a photocathode exposed to ultrafast UV pulses.
The UV pulse is generated via third harmonic generation of the output from a Ti:sapphire amplifier delivering 10$150 \mathrm{fs}$ (FWHM) pulses in the NIR. In that case, the generated UV can support an electron bunch shorter than 90 fs. As described in the previous sections, we are planning to use a Yb-based pump laser system to run the $\mathrm{THz}$ based accelerator, but its bandwidth is limited to $\sim 3 \mathrm{~nm}$, supporting $\sim 500$ fs pulses. In that case, fourth harmonic generation would produce $250 \mathrm{fs}$ long UV pulses, i.e., $250 \mathrm{fs}$ long bunch length, too long for acceleration with THz. To overcome that limitation, we build a two-stage OPA to generate short pulses in the NIR. OPAs not only give access to short UV pulses but also give flexibility in tuning the pulse spectrum to optimize the generation of the electron bunches from different photocathodes.

Figure 7 shows the schematic of the two-stage OPA driven by a fraction $(214 \mu \mathrm{J})$ of the output of the $\mathrm{Yb}: \mathrm{KYW}$ regenerative amplifier system described in Section 4.2. The pump beam is divided into two arms in order to generate a white-light continuum as seed and second harmonic as pump for the OPAs. Both OPA stages are implemented in type I phase matching configuration. $34-\mu \mathrm{J}$ pulse energy is used to generate the white light inside a $6 \mathrm{~mm}$ YAG crystal. The rest is frequency doubled with $38 \%$ conversion efficiency into a $1 \mathrm{~mm}$ thick BBO crystal resulting in $68-\mu \mathrm{J}$ pulses at $515 \mathrm{~nm}$.

The generated seed is amplified in the first OPA stage (OPA1) to $3.3 \mu \mathrm{J}$ with $13.5 \mu \mathrm{J}$ incidence pump energy. As the gain medium, a $2.5 \mathrm{~mm}$ long BBO is used. The amplified spectrum is centered around $758 \mathrm{~nm}$ with $97 \mathrm{~nm}$ bandwidth (FHWM) as shown in Figure 8(a). This output is sent through a prism compressor to have flexibility in finetuning (i) the seed dispersion and (ii) the spectral bandwidth for the second stage. This spectral narrowing is implemented by blocking the highest and lowest spectral components with knives. The pulses are then amplified to $12.3 \mu \mathrm{J}$ via the second stage OPA (OPA2) by using a $5 \mathrm{~mm}$ long BBO with $54.5-\mu \mathrm{J}$ pump pulses. The amplified pulses have a spectrum centered at $740 \mathrm{~nm}$ with a bandwidth of $25 \mathrm{~nm}$, which supports a duration shorter than $30 \mathrm{fs}$. The pulses are finally compressed by using a second prism compressor 

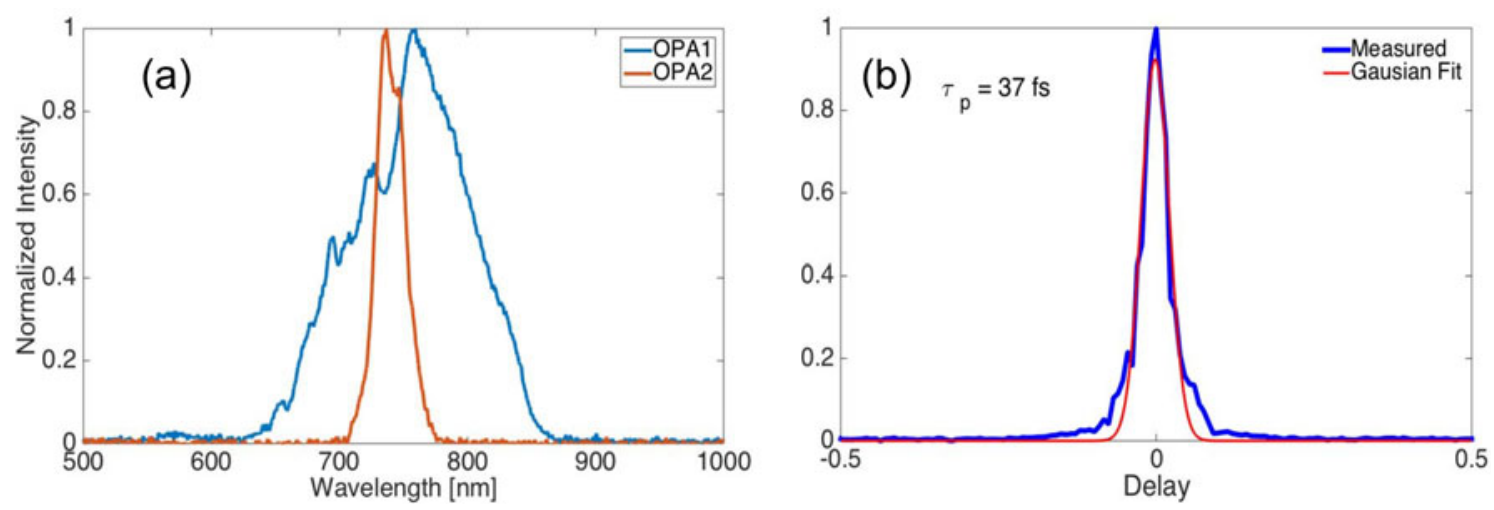

Figure 8. (a) Spectra of the first and second OPA stages (OPA1 and OPA2). (b) Autocorrelation trace of the second OPA stage after the prism compressor and the corresponding Gaussian fit.

Table 4. Summary of the pulse parameters after each module of the CT Yb:YAG laser chain.

\begin{tabular}{|c|c|c|c|c|c|c|c|c|c|}
\hline & Osc & Stretch & Regen & Tel I & Cryo I & Tel II & Cryo II & Transport & Compr \\
\hline \multirow[t]{2}{*}{$\overline{f_{\text {rep }}}$} & $70 \mathrm{MHz}$ & $70 \mathrm{MHz}$ & $1 \mathrm{kHz}$ & $1 \mathrm{kHz}$ & $100 \mathrm{~Hz}$ & $(1 \mathrm{kHz})$ & $(1 \mathrm{kHz})$ & $(1 \mathrm{kHz})$ & $(1 \mathrm{kHz})$ \\
\hline & & & & & & $100 \mathrm{~Hz}$ & $100 \mathrm{~Hz}$ & $100 \mathrm{~Hz}$ & $100 \mathrm{~Hz}$ \\
\hline$\lambda_{0}$ & & & & & $29.5 \mathrm{~nm}$ & & & & \\
\hline$\Delta \lambda$ & $5-10 \mathrm{~nm}$ & $\sim 2-3 \mathrm{~nm}$ & $2-2.5 \mathrm{~nm}$ & $2-2.5 \mathrm{~nm}$ & $0.5 \mathrm{~nm}$ & $0.5 \mathrm{~nm}$ & $0.3 \mathrm{~nm}$ & $0.3 \mathrm{~nm}$ & $0.3 \mathrm{~nm}$ \\
\hline $\mathrm{E}$ & $\sim \mathrm{nJ}$ & $\sim \mathrm{nJ}$ & $5 \mathrm{~mJ}$ & $5 \mathrm{~mJ}$ & $100 \mathrm{~mJ}$ & $100 \mathrm{~mJ}$ & $1.2 \mathrm{~J}$ & $1.2 \mathrm{~J}$ & $1 \mathrm{~J}$ \\
\hline$\tau$ & $<200 \mathrm{fs}$ & $1.6-2.4 \mathrm{~ns}$ & $1.6-2 \mathrm{~ns}$ & $0.4 \mathrm{~ns}$ & $0.4 \mathrm{~ns}$ & $0.4 \mathrm{~ns}$ & $0.4 \mathrm{~ns}$ & $0.4 \mathrm{~ns}$ & $4 \mathrm{ps}$ \\
\hline B & & & $<1.5$ & $<0.001$ & 0.485 & 0.013 & 0.489 & 0.148 & \\
\hline
\end{tabular}

down to $37 \mathrm{fs}$. The autocorrelation trace of the compressed pulses is shown in Figure 8(b).

After compression, the pulse energy dropped to $9.7 \mu \mathrm{J}$ due to transport losses and imaging optics.

In order to generate UV pulses via third harmonic generation, the compressed output of the second OPA is focused inside types I and II phase matched BBO crystals, respectively. The first crystal with $100-\mu \mathrm{m}$ thickness generates $1.5 \mu \mathrm{J}$ energetic blue pulses with $15 \%$ efficiency. After the second, $50 \mu \mathrm{m}$ thick crystal the pulse energy is measured to be $15 \mathrm{~nJ}$. This energy was required for the preliminary gun experiments, and our simulations of UV conversion show that the conversion efficiency can be improved keeping a good beam quality. The generated UV pulses are then used in electron gun experiments based on $\mathrm{THz}$ acceleration.

The following section will make use of these presented modules and build possible laser chains, before discussing the achievable pulse characteristics with each laser chain and place this in light of the requirements for driving each stage of the light source.

\section{Laser chains}

\subsection{Possible laser chains}

5.1.1. Yb:YAG cryogenically cooled laser chain A cryo-Yb:YAG laser chain starts from a dedicated output of the frontend delivering $\mathrm{nJ}$ level pulses centered at $1029.5 \mathrm{~nm}$.
After stretching, the pulses are amplified to the $\mathrm{mJ}$ level in a Yb:KYW regenerative amplifier, then to the $100 \mathrm{~mJ}$ level in a first cryo-Yb:YAG amplifier and finally to the $1 \mathrm{~J}$ level in a second cryo-Yb:YAG amplifier. The pulses are finally compressed with a Treacy compressor.

Between the amplifier modules, telescopes allow for adaptation of the beam size while relay-imaging the output of one module to the input of the next one. After the first booster amplifier, up to $100 \mathrm{~mJ}$, it is critical to strictly image the super-Gaussian near field from the output of one module to the entrance of the next one. After amplification to $1 \mathrm{~J}$, the beam profile is highly super-Gaussian. We are evaluating whether the beam will be propagated over a long distance to the compression stage to use the bell-shape far field, or whether relay-imaging telescopes will be used before and after the compressor.

Table 4 summarizes the pulse parameters after each module, such as energy $E$, spectral bandwidth $\Delta \lambda$, central wavelength $\lambda_{0}$, pulse duration $\tau$, repetition rate $f_{\text {rep }}$ and B-integral $B$.

\subsubsection{RT Yb:YAG laser chain}

The RT-Yb:YAG regenerative amplifier described in Section 4.3 is able to amplify $\mathrm{nJ}$ stretched pulses to the $100 \mathrm{~mJ}$ level, either at $1 \mathrm{kHz}$ or at $100 \mathrm{~Hz}$ depending on the thermal management. An amplifier chain based on RT-Yb:YAG consists in stretching the pulses out of an oscillator, then amplifying them in the regenerative amplifier, and finally compressing them. The parameters at the output of each 
Table 5. Summary of the pulse parameters after each module of the RT-Yb:YAG laser chain.

\begin{tabular}{|c|c|c|c|c|}
\hline & Osc & Stretch & Regen & Compr \\
\hline \multirow[t]{2}{*}{$f_{\text {rep }}$} & $70 \mathrm{MHz}$ & $70 \mathrm{MHz}$ & $1 \mathrm{kHz}$ & $(1 \mathrm{kHz})$ \\
\hline & & & $100 \mathrm{~Hz}$ & $100 \mathrm{~Hz}$ \\
\hline$\lambda_{0}$ & \multicolumn{4}{|c|}{$1030 \mathrm{~nm}$} \\
\hline$\Delta \lambda$ & $5-10 \mathrm{~nm}$ & $\sim 5 \mathrm{~nm}$ & $2 \mathrm{~nm}$ & $2 \mathrm{~nm}$ \\
\hline E & $\sim \mathrm{nJ}$ & $\sim \mathrm{nJ}$ & $130 \mathrm{~mJ}$ & $100 \mathrm{~mJ}$ \\
\hline$\tau$ & $<200 \mathrm{fs}$ & $3.25 \mathrm{~ns}$ & $1.3 \mathrm{~ns}$ & $1-2 \mathrm{ps}$ \\
\hline
\end{tabular}

Table 6. Summary of the pulse parameters after each module of the CT-Yb:YLF laser chain.

\begin{tabular}{lllrll}
\hline & Osc & Stretch & Regen & Cryo I & Compr \\
\hline$f_{\text {rep }}$ & $70 \mathrm{MHz}$ & $70 \mathrm{MHz}$ & $100 \mathrm{~Hz}$ & $100 \mathrm{~Hz}$ & $100 \mathrm{~Hz}$ \\
$\lambda_{0}$ & & & $1020 \mathrm{~nm}$ & & \\
$\Delta \lambda$ & $5-10 \mathrm{~nm}$ & $\sim 2-3 \mathrm{~nm}$ & $2.1 \mathrm{~nm}$ & $2 \mathrm{~nm}$ & $2 \mathrm{~nm}$ \\
$\mathrm{E}$ & $\sim \mathrm{nJ}$ & $\sim \mathrm{nJ}$ & $10 \mathrm{~mJ}$ & $100 \mathrm{~mJ}$ & $70 \mathrm{~mJ}$ \\
$\tau$ & $<200 \mathrm{fs}$ & $\sim 0.7-1 \mathrm{~ns}$ & $0.7 \mathrm{~ns}$ & $0.66 \mathrm{~ns}$ & $750 \mathrm{fs}$ \\
\hline
\end{tabular}

stage are summarized in Table 5. Since the spatial profile after the regenerative amplifier is expected to be Gaussian, such as in Ref. [40], the compressor might be set up with neither relay imaging nor propagation up to the far field.

\subsubsection{Yb:YLF cryogenically cooled laser chain}

The cryo-Yb:YLF detailed in Section 4.5 is able to amplify a nJ seed up to the $100 \mathrm{~mJ}$ level. This technology is limited to this energy level because of material and thermal management. Table 6 summarizes the main parameters after each stage of this laser chain. The main advantage is the low accumulation of nonlinearities (B-integral). The system is limited to $100 \mathrm{~Hz}$ and cannot be scaled up to $1 \mathrm{kHz}$ due to thermal management. Due to the near-Gaussian beam shape, no telescopes for relay imaging of the beam during transportation are necessary.

\subsection{Comparison of the achievable specifications}

The cryo-Yb:YAG laser chain will deliver pulses with $0.3 \mathrm{~nm}$ bandwidth sustaining the generation of $100 \mathrm{GHz}$ radiation, whereas the pulses out of the cryo-Yb:YLF and RT-Yb:YAG laser chains keep enough bandwidth to sustain the generation of $300 \mathrm{GHz}$ radiation.

From these considerations on the achievable pulse width and pulse energy with different laser media, from the tradeoff on the efficiency of the $\mathrm{THz}$ conversion (Section 2), and from the required $\mathrm{THz}$ energy for electron acceleration, we conclude that either a narrow-band, joule-class laser system for $100 \mathrm{GHz}$ generation or a $100 \mathrm{~mJ}$ class laser system with bandwidth sustaining the generation of $300 \mathrm{GHz}$ radiation will allow the $\mathrm{THz}$ generation required for the AXSIS project. These systems are either a $1 \mathrm{~J}$, cryo-Yb:YAG system, or a $100 \mathrm{~mJ}$ RT-Yb:YAG or cryo-Yb:YLF system.
The conversion into $\mathrm{THz}$ radiation of a $100 \mathrm{~mJ}$ level RTYb:YAG or cryo-Yb:YLF laser system should lead to $\mathrm{mJ}$ level single-cycle $\mathrm{THz}$ pulses as required for the gun.

Using the $1 \mathrm{~J} \mathrm{Yb}$ :YAG cryogenically cooled laser chain allows for generating 5 to $15 \mathrm{~mJ}$ of $\mathrm{THz}$ radiation for driving the LINAC, which can realize an energy gain to relativistic energies in the range of 5-15 MeV. With all laser chains, the highest photon energy achievable from the ICS interaction is in the range of $6 \mathrm{keV}^{[55]}$. The difference between the laser drivers occurs in the photon yield. In the ICS driven by the Yb:YLF line, we expect a yield of $10^{6}$ photons/s instead of $10^{7}$ photons/s as would be with a $1 \mathrm{~J}$ laser driver (first concept).

The data acquisition constraints during scientific experiments make a repetition rate of $1 \mathrm{kHz}$ desirable. A long-term stable and reliable $1 \mathrm{~J}, 1 \mathrm{kHz}$ laser system and a $100 \mathrm{~mJ}$ level cryo-Yb:YLF are however challenging to engineer. One remedy is the operation in a first step at $100 \mathrm{~Hz}$ repetition rate, which decreases the heat load on the crystals by a factor of 10. At the $100 \mathrm{~mJ}$ level, Yb:YAG and Yb:YLF laser amplifiers are demonstrated in our group operating at $\mathrm{CT}^{[32,33]}$ and in other groups at $\mathrm{RT}^{[41,44,45]}$. Yb:YLF at $\mathrm{CT}$ and $\mathrm{Yb}$ :YAG at RT are both good candidates for experimenting the $\mathrm{THz}$ generation and exploring electron acceleration while a $1 \mathrm{~J}$ laser system is being developed. As the conversion efficiency into $\mathrm{THz}$ radiation increases with the THz frequency, it is advantageous to implement the cryo-Yb:YLF or RT-Yb:YAG laser chains during the time frame of our project, while the $1 \mathrm{~J}$ laser systems are being developed for further upgrade. With laser chains on the level of $100 \mathrm{~mJ}$ pulse energy, it is possible to demonstrate electron acceleration and X-ray photon emission, though at lower photon energies than expected in the final machine.

The three possible laser chains exhibit an energy stability of $1 \%-2 \% \mathrm{rms}$, which is one order of magnitude larger than the requirement. For ultimate stability of the X-ray pulses, active stabilization of the IR-pulse energy will be implemented.

\section{Challenges}

\subsection{Synchronization}

Synchronization between the individual laser lines will have a significant influence on the achievable performance of the light source. Specifically, the laser systems need to be synchronized in such a way that the light-electron interactions along the machine are synchronized, too. For example, these interaction points include photo-electron generation at photocathode, terahertz electron acceleration in the gun and LINAC as well as electron interaction with the ICS laser pulse. Ideally, synchronization between the electrons and the individual $\mathrm{THz}$ or laser beams will be employed. Methods to directly observe the arrival time of electron bunches are 

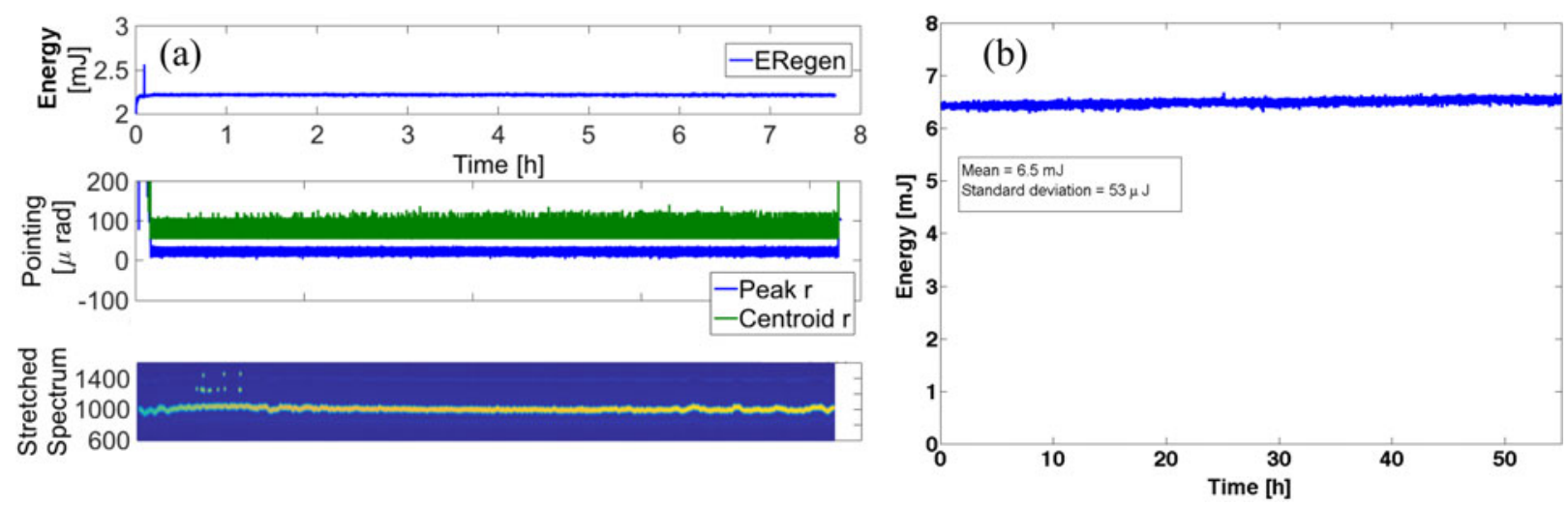

Figure 9. (a) Simultaneous measurement of the energy at the output of the Yb:KYW regenerative amplifier, pointing measured after the regenerative amplifier, and stretched spectrum. Only a fraction of the energy of the regenerative amplifier is measured without rescaling to the total energy. An rms value for the relative energy fluctuations of $0.8 \%$ is measured. The stretched spectrum was measured with a $12.5 \mathrm{GHz}$ photodiode and a $4 \mathrm{GHz}$ oscilloscope. (b) Long term measurement of the $\mathrm{Yb}: \mathrm{KYW}$ regenerative amplifier output.

under development ${ }^{[56-58]}$ but will be discussed elsewhere. In the following, we will describe our approach to lock the individual laser beams prior to $\mathrm{THz}$ or laser-electron interaction.

Considering the multiple round trips within the regenerative amplifiers and the multi-pass amplifiers a total optical path in excess of $300 \mathrm{~m}$ is expected for each beam line, despite the fact that the physical separation of the seed source and the accelerator is only about $10 \mathrm{~m}$. Assuming a typical thermal expansion of the materials used to house the laser of approximately $\alpha=10^{-5} \mathrm{~K}^{-1}$, a thermal timing drift of about $10 \mathrm{ps} / \mathrm{K}$ is expected. In addition, fast, near random, timing jitter is expected due to vibrations caused by acoustic waves that will also be on the order of a few hundreds of femtoseconds. Because of this large expected timing error a prelock between individual lasers and/or THz sources should be established along the propagation of the laser beams as a precursor for full $\mathrm{THz}$ to electron synchronization.

In order to achieve a manageable engineering problem, multiple levels of accuracy are considered. The first level provides timing on a timescale of the seed laser repetition rate. By electronically selecting a pulse for separate beam lines, path length differences can be corrected on multiples of the seed repetition rate.

In the second synchronization level, optical path lengths must be length matched for all beam lines derived from the same low-repetition-rate source, e.g., a regenerative amplifier. Optical pulses with a length on the order of a few picoseconds can be well synchronized using balanced optical cross-correlators (BOCs) as timing jitter detectors ${ }^{[59,60]}$. To employ this method, one pulse is declared a master pulse, preferentially the pulse arriving at the electron gun. Copropagating this pulse with the electron bunch and adding slight delays using high precision delay lines creates a timescale on the same total length as the accelerator. BOCs can then detect timing deviations and corrections can be made at the beginning of the individual beam lines.
A third level of locking can be made after the THz generation by combining techniques from the standard optical BOC and electro-optical sampling of $\mathrm{THz}$ waveforms. The optical gun pulse is used as master, and that pulse is used to determine the arrival time of a $\mathrm{THz}$ pulse close to the accelerator. This is especially advantageous for the multicycle $\mathrm{THz}$ pulses where the phase between the $\mathrm{THz}$ and the electron bunch is of higher importance than the envelope and the bunch ${ }^{[61]}$.

Independent of the method to achieve synchronization, the low final repetition rate results in a severe limitation of achievable performance. The laser pulses sample the acoustic and thermal noise with 100 to $1000 \mathrm{~Hz}$, downfolding higher frequencies into the Nyquist spectral range. To minimize this impact, specific attention must be paid to the noise sources during facility construction and thermal stabilization of the laser system aiming to damp vibrations with higher frequency components.

\subsection{Long-term stability}

The scientific experiments intended with the table-top X-ray source require laser operation for the time of alignment and of data acquisition, both of which may last for several hours. This sets a strong constraint on the long-term stability of the laser system and $\mathrm{THz}$ generators.

Efforts have been already set in engineering the Yb:KYW regenerative amplifier and the cryo-Yb:YAG amplifiers. Measurements on the currently available amplifiers, shown in Figure 9, feature good pointing and energy stability of the regenerative amplifier, with $50 \mu \mathrm{rad}$ and $0.8 \% \mathrm{rms}$ stability, respectively. Figure 9(a) displays also the spectral stability with a fast photodiode and oscilloscope receiving the stretched pulse at the output of the regenerative amplifier over $8 \mathrm{~h}$. After a warm-up time due to thermalization of the fiber-based stretcher containing pre-amplifiers, the signal 

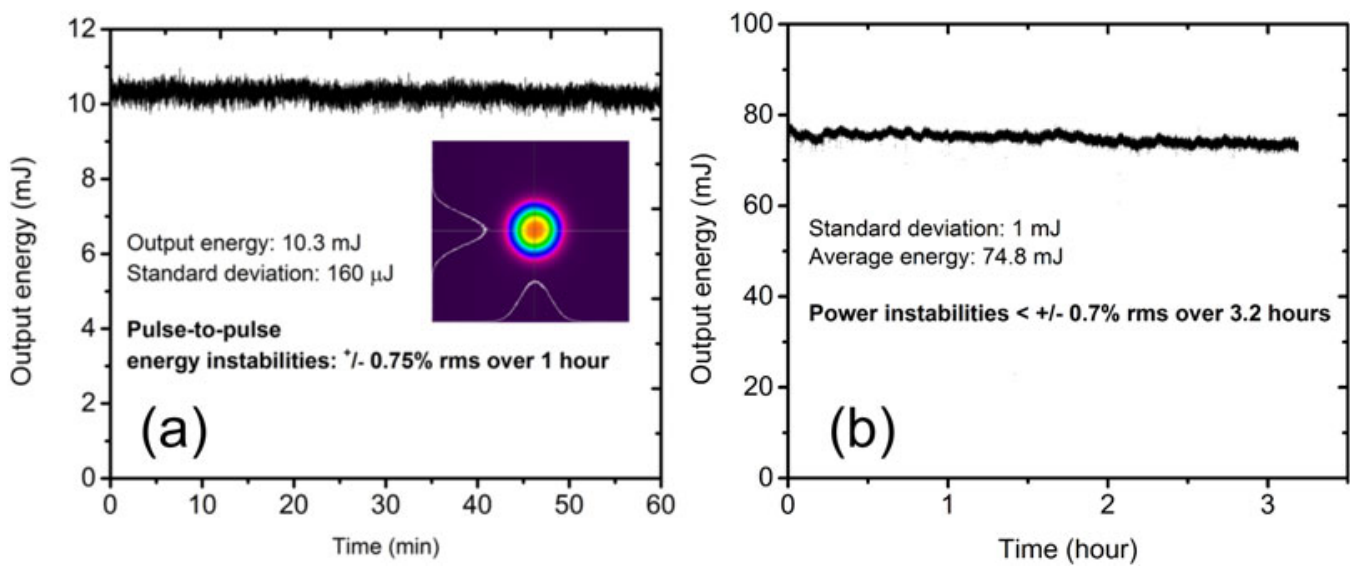

Figure 10. (a) Measured 1-h stability of the regenerative amplifier output at the $10 \mathrm{~mJ}$ energy level. The computed shot-to-shot instabilities are less than $\pm 0.75 \% \mathrm{rms}$ over $1 \mathrm{~h}$. In inset, the measured spatial intensity profile at $10 \mathrm{~mJ}$ output energy. (b) Measured output energy stability recorded over $3.5 \mathrm{~h}$ at $\sim 75 \mathrm{~mJ}$ output energy. The observable slow drift is attributed to a minor drift in seed energy of the current frontend. Energy instabilities less than $\pm 0.7 \%$ over $3.2 \mathrm{~h}$ are routinely achieved.

is constant. The warm-up time and stability have been improved by partially stabilizing the stretcher in temperature and flooding the fibers with dry nitrogen to mitigate humidity effects.

The Yb:YLF laser amplifier chain operating daily in our lab exhibits a pulse-to-pulse stability below $1 \%$ after the regenerative and booster amplifiers. Figure 10 shows the measurement stability of these two modules. The observable slow drift is attributed to a minor drift in seed energy of the currently used frontend. In the updated version of the frontend, emphasis is placed on enhanced output parameter stability. For example, it has been demonstrated that operating the fiber amplifiers behind the master oscillator in saturation significantly lowers any fluctuation of the seed energy for subsequent amplifiers (such as the regenerative amplifiers or multi-pass energy boosters) for frequencies $<1 \mathrm{kHz}$, which is the inverse of the excited state lifetime of the fiber dopant ytterbium. Additionally, environmental influences on system performance, such as temperature and humidity, are passively and actively controlled.

In all cryogenically cooled modules, automatic refill systems will be implemented to ensure long-term operation. Implementing pointing stabilizers after each module will enhance long-term operation by minimizing the influence of misalignments between stages.

The output of the OPAs for UV generation, already operating for electron acceleration experiments, demonstrates a good day-to-day stability. The pulse energy measurement exhibits very good stability over $15 \mathrm{~h}$ as shown in Figure 11. The average pulse energy is measured to be $9.7 \mu \mathrm{J}$ with $2.9 \%$ standard deviation. Final stability of the output beam depends strongly on the individual performance of the nonlinear stages. The sequence of multiple, not-all saturated stages can easily lead to an increase of the pulse-to-pulse fluctuations. UV generation is known for severe degradation

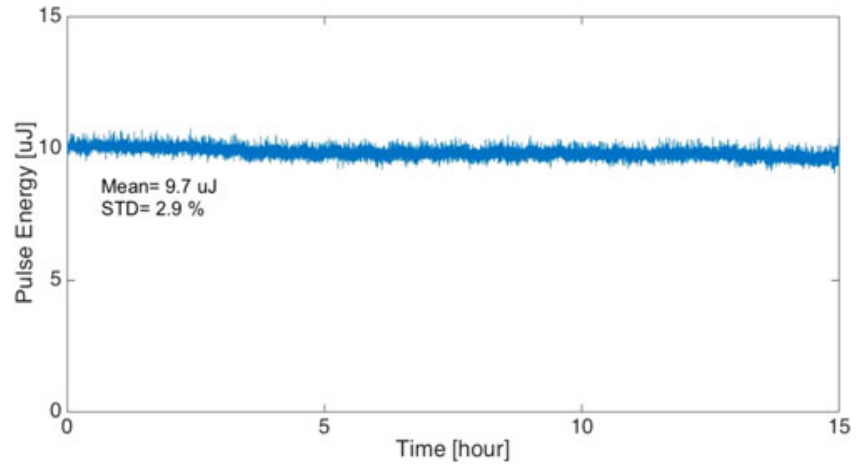

Figure 11. Pulse energy measurement of the compressed OPA output over $15 \mathrm{~h}$.

issues over time. We mitigate this issue by generating UV in the vacuum chamber of the gun, minimizing contamination and reducing transport optics.

\subsection{Controls and diagnostics}

The complex laser system requires controls and monitors to check the correct operation of each module. Table 7 summarizes the diagnostics chosen for all modules. Temperature and humidity are crucial parameters to be monitored, since they influence the total beam path, where changes might influence the pointing of the components. Optical parameters to monitor are energy, pulse duration, spectral width - after the frequency tripling into UV and the compressors to help for debugging -, and beam quality. The correct function of the laser diodes is monitored and controlled with current and temperature. The liquid nitrogen level of all cryogenically cooled modules will be similarly monitored. 
Table 7. Diagnostics for the modules.

\begin{tabular}{|c|c|c|c|c|c|c|c|c|c|}
\hline$\overline{\text { Diagnostic }}$ & Seeder & Stretcher & $\begin{array}{l}\text { Yb:KYW } \\
\text { regen }\end{array}$ & $\begin{array}{l}\text { Yb:YAG } \\
\text { CTD } 100 \mathrm{~mJ}\end{array}$ & $\begin{array}{l}\text { Yb:YAG } \\
\text { CTD } 1 \mathrm{~J}\end{array}$ & $\begin{array}{l}\text { Yb:YLF } \\
\text { Regen }\end{array}$ & $\begin{array}{l}\text { Yb:YLF } \\
\text { booster }\end{array}$ & Compressors & UV \\
\hline Power & $\mathrm{X}$ & & & & & & & & \\
\hline Energy & $\mathrm{X}$ & $\mathrm{X}$ & $\mathrm{X}$ & $\mathrm{X}$ & $\mathrm{X}$ & $\mathrm{X}$ & $\mathrm{X}$ & $\mathrm{X}$ & $\mathrm{X}$ \\
\hline Reprate & $\mathrm{X}$ & $\mathrm{X}$ & $\mathrm{X}$ & & & $\mathrm{X}$ & & & \\
\hline Temporal profile & & $\mathrm{X}$ & $\mathrm{X}$ & $\mathrm{X}$ & $\mathrm{X}$ & $\mathrm{X}$ & $\mathrm{X}$ & $\mathrm{X}$ & $X$ \\
\hline Spectrum & & & & & & & & $\mathrm{X}$ & $\mathrm{X}$ \\
\hline Beam profile & & & $\mathrm{X}$ & $\mathrm{X}$ & $\mathrm{X}$ & $\mathrm{X}$ & $\mathrm{X}$ & $\mathrm{X}$ & $\mathrm{X}$ \\
\hline Currents & $\mathrm{X}$ & $\mathrm{X}$ & $\mathrm{X}$ & & & $\mathrm{X}$ & $\mathrm{X}$ & & \\
\hline Diode and crystal temperatures & $\mathrm{X}$ & $\mathrm{X}$ & $\mathrm{X}$ & & & $\mathrm{X}$ & $X$ & & \\
\hline $\begin{array}{l}\text { Diode power before and after } \\
\text { crystal }\end{array}$ & & & & $\mathrm{X}$ & $\mathrm{X}$ & & & & \\
\hline Temperature & $\mathrm{X}$ & $\mathrm{X}$ & $\mathrm{X}$ & $\mathrm{X}$ & $\mathrm{X}$ & $\mathrm{X}$ & $X$ & $\mathrm{X}$ & \\
\hline Humidity & $\mathrm{X}$ & $\mathrm{X}$ & $\mathrm{X}$ & $\mathrm{X}$ & $\mathrm{X}$ & $\mathrm{X}$ & $\mathrm{X}$ & $\mathrm{X}$ & \\
\hline LN2 level & & & & $\mathrm{X}$ & $\mathrm{X}$ & $\mathrm{X}$ & $X$ & & \\
\hline Chiller & & & $\mathrm{X}$ & $\mathrm{X}$ & $\mathrm{X}$ & $\mathrm{X}$ & $X$ & & \\
\hline Vacuum & & & & $X$ & $\mathrm{X}$ & $\mathrm{X}$ & $\mathrm{X}$ & & \\
\hline
\end{tabular}

\subsection{Cryogenic cooling}

The cryogenic cooling of the CT laser chain is enforced with liquid nitrogen for the $100 \mathrm{~mJ} \mathrm{Yb}$ :YAG and Yb:YLF amplifier modules. The LN2 consumption, measured empirically, of the $100 \mathrm{~mJ}$ and $1 \mathrm{~J}$ stage cryo-Yb:YAG amplifiers together is $132 \mathrm{~L}$ per $12 \mathrm{~h}$ at $1 \mathrm{kHz}$, whereas the cryo$\mathrm{Yb}$ :YLF regenerative amplifier requires $1.2 \mathrm{~L}$ per $6 \mathrm{~h}$ and the following 4-pass amplifier $\sim 2 \mathrm{~L}$ every $4-5 \mathrm{~h}$ at $10 \mathrm{~Hz}$. Scaled to $100 \mathrm{~Hz}$, this represents in total $\sim 75 \mathrm{~L}$ for operating the cryo-Yb:YLF at $12 \mathrm{~h}$ per day. This will be implemented with automatic refill systems. The advantage of using evaporating liquid nitrogen over closed loop compressors is the low amount of vibrations introduced into the laser system. It is worth mentioning that new compressor based cryogenic cooling systems are currently available (though with not yet enough heat removal capacity for scaling up to $1 \mathrm{kHz}$, but up to $\sim 500 \mathrm{~Hz}$ ) and under development. We are testing and experimenting with those systems. Ultimately, testing and empirical experience will allow to take a decision between liquid nitrogen cooling and compressor based systems.

\subsection{Beam transport}

For radiation protection purposes, the accelerator must be located in a shielded room, separated from the laser system. The high-energy laser beams will then be transported to the accelerator setup via chicanes needed to avoid direct line of sight from one room into the other. In principle, either the IR beams or the $\mathrm{THz}$ beams could be transported. Optics for $\mathrm{THz}$ radiation exhibit high losses and are not adequate for transport over long distances requiring multiple optics. The IR pulses, even though highly energetic, are easier to transport, whether stretched or unstretched. Considering $1 \mathrm{~J}, 5$ ps compressed pulses with
$20 \mathrm{~mm}$ flat-top beam diameter, a transport in air would accumulate $0.03 \mathrm{rad} / \mathrm{m} \mathrm{B}$-integral (assuming $n_{2 \text {,air }}=8 \times$ $10^{-24} \mathrm{~cm}^{2} / \mathrm{W}^{[62]}$, without taking the spatial ripples appearing during propagation into account), i.e., $0.6 \mathrm{rad}$ over the intended $20 \mathrm{~m}$. This does not require vacuum transport. However, as the spatial profile at the output of the amplifiers is super-Gaussian, relay-imaging telescopes will be required to image the near field to the $\mathrm{THz}$ generators. For this reason, we will use low vacuum for transportation of the beams.

\section{Overall description of the laser system}

\subsection{Presentation of three concepts}

We have developed three concepts for the laser system driving our X-ray light source, two operating with cryogenically cooled laser crystals: the first one is based on Yb:YAG whereas the second one takes advantage from both $\mathrm{Yb}$ :YAG and $\mathrm{Yb}$ :YLF. A third concept relies on RT Yb:YAG amplifiers, which are not easily scalable to the joule level, but partially commercially available.

\subsubsection{Design solution 1}

Figure 12 displays the first solution, completely based on Yb:YAG high-energy amplifiers. A common oscillator seeds the four laser chains. Each high-energy laser chain contains a stretcher, a regenerative amplifier, a booster stage up to $100 \mathrm{~mJ}$ (CTD $100 \mathrm{~mJ}$ ), a final booster up to $1 \mathrm{~J}$ (CTD $1 \mathrm{~J}$ ) and a compressor. In order to mitigate gain narrowing occurring during amplification over nine decades (from the $\mathrm{nJ}$ level to the $\mathrm{J}$ level), Yb:KYW was chosen as the gain medium of the regenerative amplifier. This implementation assures the two following high-energy amplifiers to be optimally seeded over the full gain bandwidth, delivering $0.3 \mathrm{~nm}$ wide pulses corresponding to $5 \mathrm{ps}$ after compression. The pulses are 


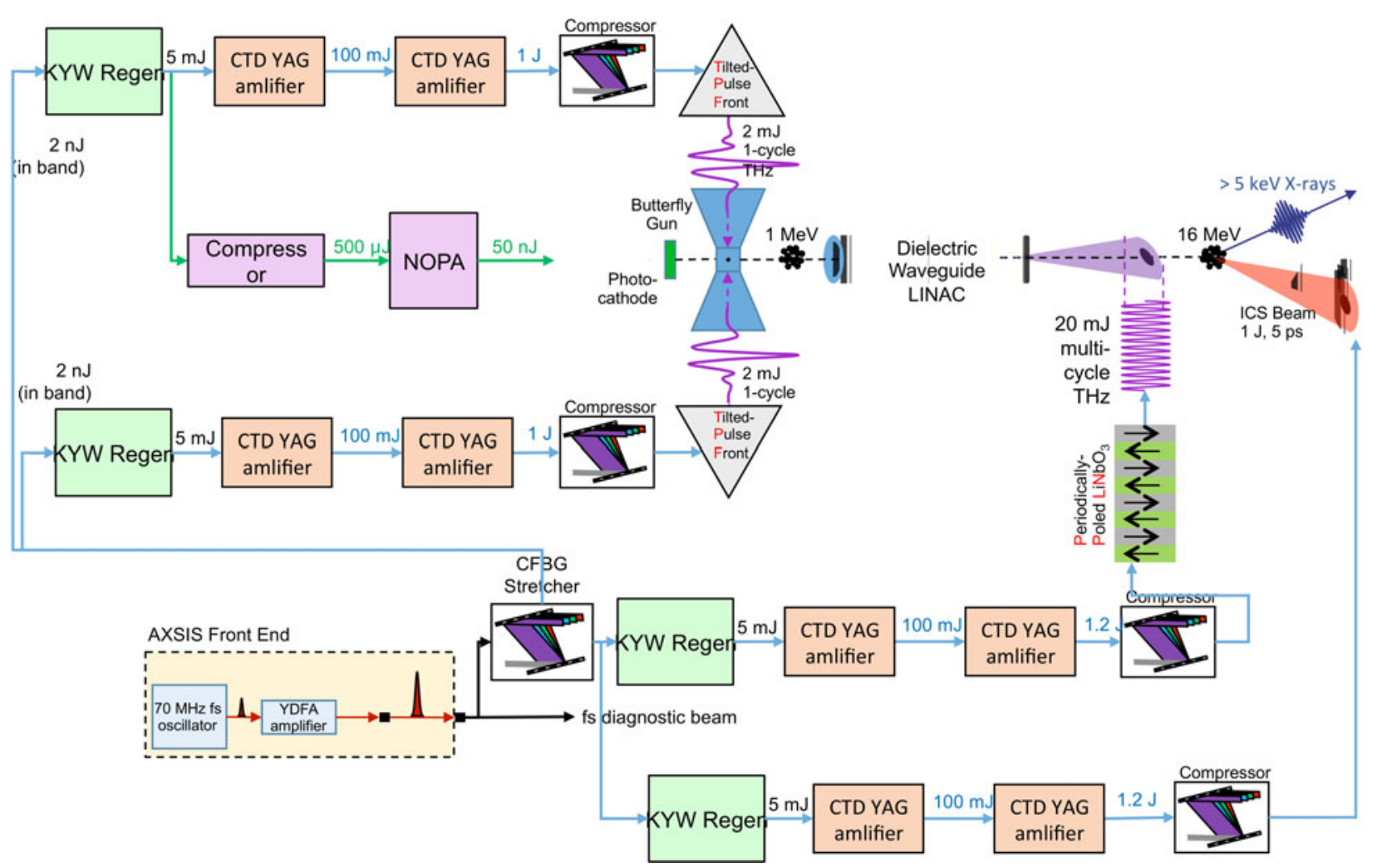

Figure 12. Schematic representation of the laser system based on cryo-Yb:YAG laser systems.

compressed close to transform-limited pulse duration if the compressors are well matched with the stretchers. UV pulses are generated from a fraction of the beam delivered by one of the Yb:KYW regenerative amplifiers. The nonlinear conversion stages for this purpose comprise white-light generation (WLG), optical parametric amplification around $759 \mathrm{~nm}$ and frequency tripling to $253 \mathrm{~nm}$. WLG requires ultra-short pulses to generate compressible pulses, where pulses well below a picosecond duration have experimentally proven to be advantageous ${ }^{[63]}$. The corresponding spectral bandwidth of several nanometers exceeds the optimal bandwidth for seeding the Yb:YAG amplifiers, meaning that a trade-off is necessary between large bandwidth for few hundreds of femtosecond long pulses for WLG and effective in-band seeding of the YAG amplifiers. This compromise has led to using $\sim 3 \mathrm{~nm}$ bandwidth, corresponding to $500 \mathrm{fs}$ (transform limited, assuming $\operatorname{sech}^{2}$ pulses).

\subsubsection{Design solution 2}

Figure 13 shows the conceptual layout of the laser machine based on Yb:YLF and Yb:YAG amplifiers. Here, three laser chains are based on $\mathrm{Yb}$ :YLF to drive the two $\mathrm{THz}$ stages for the gun and the ICS stage, and only the one laser chain for generating $\mathrm{THz}$ feeding the LINAC is based on Yb:YAG amplifiers. The frontend comprises a $70 \mathrm{MHz}$ oscillator and a distribution system to seed all the laser chains plus one output for diagnostic of the $\mathrm{THz}$ pulses.
The three Yb:YLF laser chains are split after a common stretcher and comprise a regenerative amplifier and a power amplifier boosting the energy up to $100 \mathrm{~mJ}$. The pulses are compressed with Treacy compressors to sub-picosecond duration. The Yb:YAG laser chain starts with the $\mathrm{Yb}: \mathrm{KYW}$ regenerative amplifier delivering $5 \mathrm{~mJ}, 1.5 \mathrm{~nm}$ broad pulses to seed the two-stage high-energy multi-pass cryo-Yb:YAG amplifiers. The UV pulses are generated from a fraction of the energy of one of the YLF regenerative amplifiers, compressed separately from the other chains: a white-light generator broadens the available spectrum, followed by a narrow-band optical parametric amplifier (OPA) and a frequency tripling conversion stage. The 100-200 nJ resulting pulses are spectrally centered at $253 \mathrm{~nm}$ corresponding to the work function of copper used in the photocathode and transform-limited to $20 \mathrm{fs}$. As in the previous concept, the pulses picked off from the Yb:YLF regenerative amplifier have to be compressed to transform-limited level for a proper operation of the WLG. The amplification bandwidth of Yb:YLF allows for $\sim 1.5 \mathrm{~nm}$, i.e., 800 fs. An alternative path is to use for the OPA and UV generation the compressed pulses after the power amplifier. This would have the disadvantages of first adding additional optical path length to the system, through which the stability of the system will be degraded. Another consequence of using the output of the power amplifier is the generation of white light with slightly longer pulses of $1-5 \mathrm{ps}$, which increases 


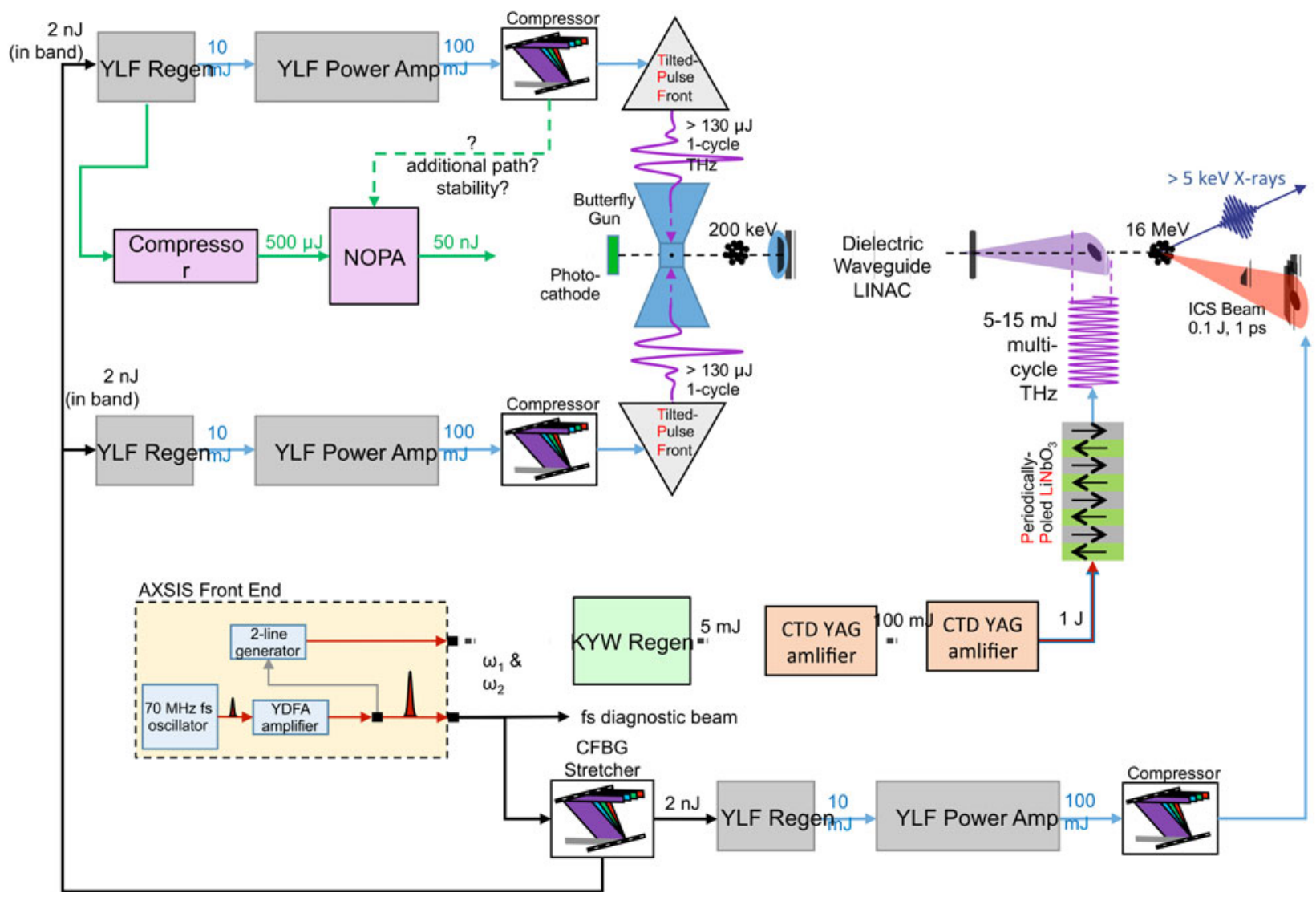

Figure 13. Schematic representation of the laser system based on cryo-Yb:YLF and cryo-Yb:YAG laser systems.

the risks in the compressibility of the pulses out of the $\mathrm{OPA}^{[63]}$. The advantage would be to avoid the necessity to match two compressors based on different technologies (cf. Section 4.6).

\subsubsection{Design solution 3}

Figure 14 shows the concept of a system completely based on Yb:YAG amplifiers operating at room temperature. Compared with the previous case, all cryogenically cooled laser systems but one amplifier would be replaced with RT Yb:YAG amplifiers. In the gun and ICS laser chains, one regenerative amplifier increases the $\sim \mathrm{nJ}$ level seed pulses up to $120 \mathrm{~mJ}$, so that after compression $100 \mathrm{~mJ}$ are available. In the LINAC laser chain though, a higher energy of $1 \mathrm{~J}$ is required to generate $\mathrm{mJ}$ level $\mathrm{THz}$ radiation. For this purpose, the joule-class cryo-Yb:YAG amplifier needs to be seeded at $100 \mathrm{~mJ}$ level by a cryo-Yb:YAG regenerative amplifier, which is itself seeded at the mJ level by a Yb:KYW regenerative amplifier. The pulses for UV generation are derived from one of the laser beams feeding the gun inputs.

In all three concepts, the synchronization of the pulses occurs at the low-energy stages, while the delay between two pulses is detected just before the $\mathrm{THz}$ frequency conversion stages.

\subsection{Discussion}

These three concepts are able to deliver pulses serving the different stages of the table-top X-ray light source. Obviously, variants of these possibilities can be thought of.

The first version is advantageous because it delivers enough optical pulse energy in all stages to achieve the full specification of the table-top X-ray light source. One regenerative amplifier could seed the two cryo-Yb:YAG lines for the gun in order to minimize the jitter between the two gun drivers.

The second and third versions mitigate this risk by requiring only 1-J class amplifier, whose output pulses do not require compression, thus avoiding the issues coming along the compression of high-energy, flat-top beams. The cryoYb:YLF laser amplifiers have not yet been demonstrated at $100 \mathrm{~Hz}$, compared to the RT-Yb:YAG amplifiers. However, the performances of the RT-Yb:YAG amplifiers in terms of pulse energy, repetition rate, beam quality and daily operation and maintenance (without use of liquid nitrogen) make the third version highly attractive. Scaling cryogenic Yb:YLF laser systems to high average power has been demonstrated up to $100 \mathrm{~W}(10 \mathrm{kHz}, 10 \mathrm{~mJ})$ average power when operated in a CPA architecture ${ }^{[64]}$. These systems could operate at high average power since the relatively 


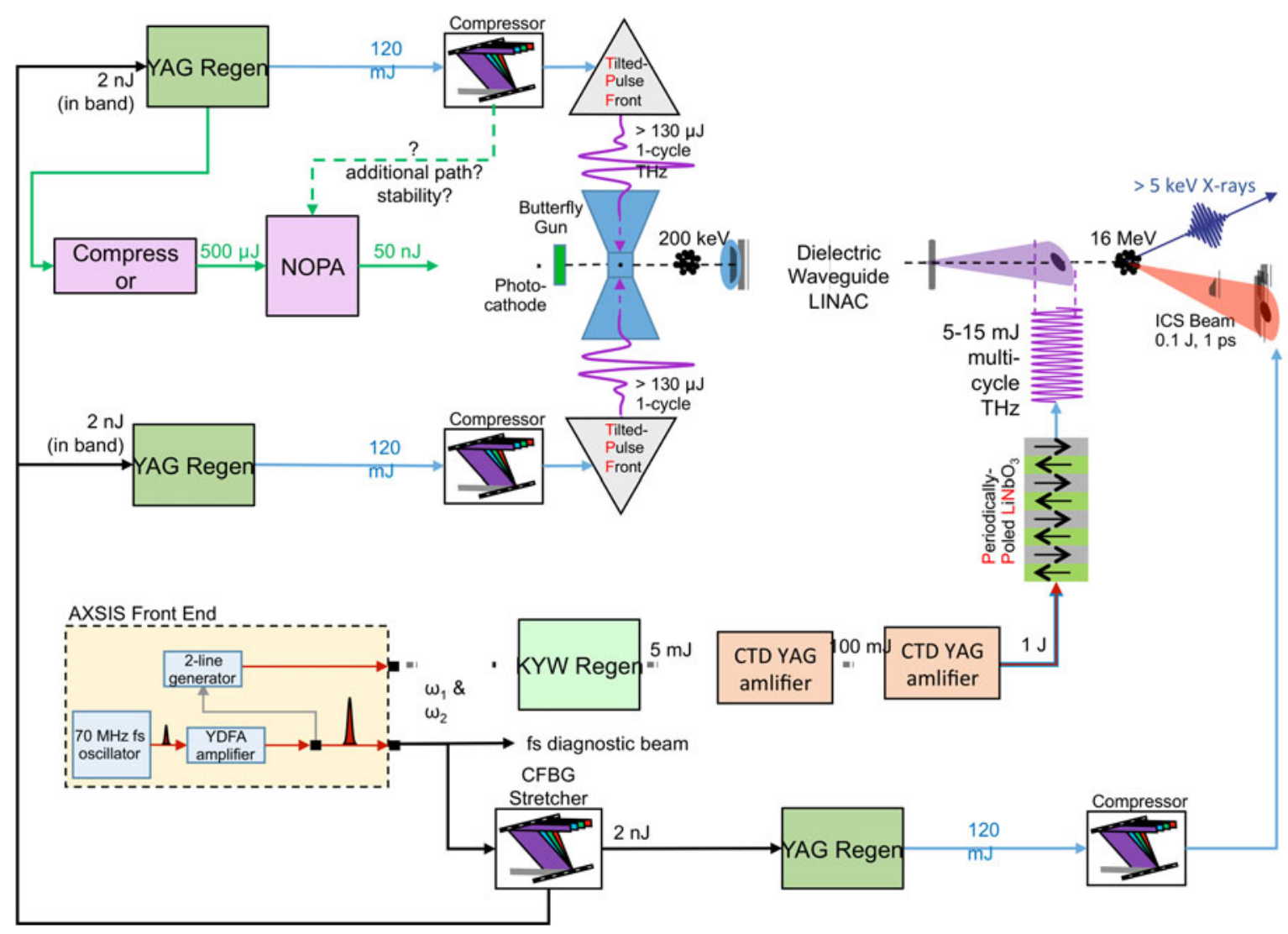

Figure 14. Schematic representation of the laser system based on RT-Yb:YAG laser systems.

low energy enables tight focusing of the pump therefore enabling high gain. In the high-energy regime, the pump area must be increased in order to ensure that the signal fluence remains below the damage fluence, which ultimately reduces the gain or demands increased pump levels (with a direct impact on thermal loading of the gain medium). There are two solutions to untie this dilemma which consists in increasing the signal pulse duration to the ns to multins regime - a measure we have already implemented and that allows maintaining relatively small pump area and therefore acceptable gain levels - or resorting to regenerative type amplification where low gain can be compensated for by increasing the number of passes in the gain medium. Operation in the $\mathrm{kHz}$ regime will require merging these two approaches and operating any regenerative type amplifier at one of the stability points where period doubling can be avoided $^{[65]}$.

In all versions, the multi-cycle, multi-mJ level $\mathrm{THz}$ stage for the LINAC is driven by a 1-J-class laser, here presented with a sequence of a $\mathrm{Yb}: \mathrm{KYW}$ regenerative amplifier and cryo-Yb:YAG amplifier seeding the high-energy cryoYb:YAG amplifier. The Yb:KYW amplifier restricts the variations in bandwidth and the spectral narrowing during six decades of amplification. One could replace the first cryoYb:YAG amplifier with an RT-Yb:YAG amplifier, for ease of maintenance, similarity with the other laser chains and decrease of engineering effort; however, the spectral peak of amplification shifts slightly between RT and CT operations.

Concerning the UV generation, the first version is advantageous for the bandwidth offered by the $\mathrm{Yb}: \mathrm{KYW}$ regenerative amplifier. However stable WLG has been demonstrated with ps-long pulses ${ }^{[63,66,67]}$.

One last point to discuss is the requirements induced by the laser system on the facility.

\subsection{Facility requirements}

Figure 15 shows the layout of two cryo-Yb:YAG laser chains symmetrically built on one table. For the laser system based on cryo-Yb:YAG only, three such tables would be required. Here, the compressors are assumed to be built close to the $\mathrm{THz}$ generators, after beam transportation into the radiation safe room. Two racks comprising the control electronics and the diagnostics serve each laser chain. A similar table layout can be made for the cryo-Yb:YLF based laser system.

In total, the laser system necessitates a lab of roughly $190 \mathrm{~m}^{2}$. The total electrical power is $\sim 46 \mathrm{~kW}$ in the case of all cryo-Yb:YAG laser systems (given $11 \mathrm{~kW}$ for one chain of $100 \mathrm{~mJ}$ and $1 \mathrm{~J}$ amplifiers), decreasing to $32 \mathrm{~kW}$ for the 


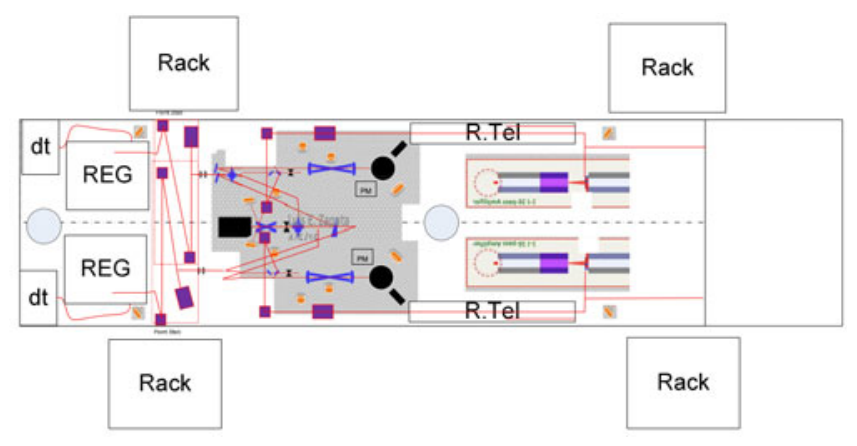

Figure 15. Layout of two Yb:YAG laser chains on one optical table. The seed pulses are fiber delivered. The delay stage (dt) is followed by the Yb:KYW regenerative amplifier (REG), followed by the two CTD amplifiers with a relay imaging telescope (R.Tel) in between. After the regenerative amplifier and the first CTD amplifier, there is a pointing stabilizer. The spatial profile of the beam is measured after each stage. The alignment laser for first alignment of the $100 \mathrm{~mJ}$ CTD is represented.

cryo-Yb:YLF/cryo-Yb:YAG system (given $6.7 \mathrm{~kW} /$ chain for the cryo-Yb:YLF at $100 \mathrm{~Hz}$ ). This decrease is due to the lower output energy of the cryo-Yb:YLF systems compared to the $1 \mathrm{~J}$ output energy of the cryo-Yb:YAG systems: the laser diodes require only $19 \mathrm{~kW}$ electrical power instead of $44 \mathrm{~kW}$. In the case of the RT-Yb:YAG system, the electrical consumption amounts to $40 \mathrm{~kW}$ (given $9.4 \mathrm{~kW} /$ chain for the RT-Yb:YAG amplifiers). The electrical power, which is neither converted into optical power nor removed via direct water and LN2 cooling, will be removed through the water cooling of the racks.

\section{Conclusion}

The conceptual design of a laser system driving a table-top, THz-driven X-ray source was discussed. The laser system generates several outputs to drive successive stages of the light source. First, the specifications for these outputs were inferred from each specific task. After discussing the achievable laser performances with cryo-Yb:YAG, cryo-Yb:YLF and RT-Yb:YAG, laser amplifier modules are presented, with the available experimental results. These performances and results are then discussed in light of each task. We then envisage and compare three architectures, one based on all cryo-Yb:YAG amplifiers, the other one based on cryo-Yb:YAG and cryo-Yb:YLF laser chains and the third one based on RT-Yb:YAG laser chains. The cryo-Yb:YLF based one has for advantage that the lasers have been demonstrated in our laboratory, though at lower repetition rate over long time periods. The RT-Yb:YAG, though not yet demonstrated in our group, seems to be the most promising and straightforward solution in terms of daily maintenance and scaling to high repetition rates. In all cases, a Jclass amplifier currently under development will drive the multi-cycle THz stage for LINAC. Engineering challenges such as synchronization, long-term operation, stability, beam transport and facility requirements are discussed.

The authors declare no conflict of interest.

\section{Acknowledgements}

This work has been supported by the European Research Council under the European Union's Seventh Framework Programme (FP/2007-2013)/ERC Grant Agreement n. 609920 and the excellence cluster 'The Hamburg Centre for Ultrafast Imaging-Structure, Dynamics and Control of Matter at the Atomic Scale' of the Deutsche Forschungsgemeinschaft. Dr. Calendron acknowledges the support by a Helmholtz Postdoctoral grant. The authors gratefully acknowledge helpful discussions with the engineering group, Thomas Tilp, Matthias Schutz and Andrej Berg.

\section{References}

1. S. Kühn, M. Dumergue, S. Kahaly, S. Mondal, M. Füle, T. Csizmadia, B. Farkas, B. Major, Z. Várallyay, F. Calegari, M. Devetta, F. Frassetto, E. Månsson, L. Poletto, S. Stagira, C. Vozzi, M. Nisoli, P. Rudawski, S. Maclot, F. Campi, H. Wikmark, C. L. Arnold, C. M. Heyl, P. Johnsson, A. L'Huillier, R. Lopez-Martens, S. Haessler, M. Bocoum, F. Boehle, A. Vernier, G. Iaquaniello, E. Skantzakis, N. Papadakis, C. Kalpouzos, P. Tzallas, F. Lépine, D. Charalambidis, K. Varjú, K. Osvay, and G. Sansone, J. Phys. B 50, 132002 (2017).

2. A. R. Maier, M. Kirchen, and F. Grüner, in Synchrotron Light Sources and Free-Electron Lasers: Accelerator Physics, Instrumentation and Science Applications (Springer International Publishing, 2016), p. 1.

3. K. W. D. Ledingham, W. Galster, and R. Sauerbrey, Br. J. Radiol. 80, 855 (2007).

4. R. Prasad, S. Ter-Avetisyan, D. Doria, K. E. Quinn, L. Romagnani, P. S. Foster, C. M. Brenner, J. S. Green, P. Gallegos, M. J. V. Streeter, D. C. Carroll, O. Tresca, N. P. Dover, C. A. J. Palmer, J. Schreiber, D. Neely, Z. Najmudin, P. McKenna, M. Zepf, and M. Borghesi, Nucl. Instrum. Methods Phys. Res. A 653, 113 (2011).

5. F. X. Kärtner, F. Ahr, A.-L. Calendron, H. Cankaya, S. Carbajo, G. Chang, G. Cirmi, K. Dörner, U. Dorda, A. Fallahi, T. Hartin, M. Hemmer, R. Hobbs, Y. Hua, R. Huang, R. Letrun, N. Matlis, V. Mazalova, O. Mücke, E. Nanni, W. Putnam, K. Ravi, F. Reichert, I. Sarrou, X. Wu, H. Ye, L. Zapata, D. Zhang, C. Zhou, R. J. D. Miller, K. Berggren, H. Graafsma, A. Meents, R. W. Assmann, H. N. Chapman, and P. M.-L. Fromme, in NIMA Proceedings (2016).

6. J. M. J. Madey, J. Appl. Phys. 42, 1906 (1971).

7. A. Aquila, M. S. Hunter, R. B. Doak, R. A. Kirian, P. Fromme, T. A. White, J. Andreasson, D. Arnlund, S. Bajt, T. R. M. Barends, M. Barthelmess, M. J. Bogan, C. Bostedt, H. Bottin, J. D. Bozek, C. Caleman, N. Coppola, J. Davidsson, D. P. DePonte, V. Elser, S. W. Epp, B. Erk, H. Fleckenstein, L. Foucar, M. Frank, R. Fromme, H. Graafsma, I. Grotjohann, L. Gumprecht, J. Hajdu, C. Y. Hampton, A. Hartmann, R. Hartmann, S. Hau-Riege, G. Hauser, H. Hirsemann, P. Holl, J. M. Holton, A. Hömke, L. Johansson, N. Kimmel, S. Kassemeyer, F. Krasniqi, K.-U. Kühnel, M. Liang, L. Lomb, 
E. Malmerberg, S. Marchesini, A. V. Martin, F. R. N. C. Maia, M. Messerschmidt, K. Nass, C. Reich, R. Neutze, D. Rolles, B. Rudek, A. Rudenko, I. Schlichting, C. Schmidt, K. E. Schmidt, J. Schulz, M. M. Seibert, R. L. Shoeman, R. Sierra, H. Soltau, D. Starodub, F. Stellato, S. Stern, L. Strüder, N. Timneanu, J. Ullrich, X. Wang, G. J. Williams, G. Weidenspointner, U. Weierstall, C. Wunderer, A. Barty, J. C. H. Spence, and H. N. Chapman, Opt. Express 20, 2706 (2012).

8. S. P. Hau-Riege, Phys. Rev. Lett. 108, 238101 (2012).

9. J. B. Rosenzweig, D. B. Cline, R. N. Dexter, D. J. Larson, A. W. Leonard, K. R. Mengelt, J. C. Sprott, F. E. Mills, and F. T. Cole, Aip Conf. Proc. 130, 226 (1985).

10. J. B. Rosenzweig, D. B. Cline, B. Cole, H. Figueroa, W. Gai, R. Konecny, J. Norem, P. Schoessow, and J. Simpson, Phys. Rev. Lett. 61, 98 (1988).

11. E. Esarey, C. B. Schroeder, and W. P. Leemans, Rev. Mod. Phys. 81, 1229 (2009).

12. N. D. Powers, I. Ghebregziabher, G. Golovin, C. Liu, S. Chen, S. Banerjee, J. Zhang, and D. P. Umstadter, Nat. Photon. 8, 28 (2014).

13. S. Kneip, C. McGuffey, J. L. Martins, S. F. Martins, C. Bellei, V. Chvykov, F. Dollar, R. Fonseca, C. Huntington, G. Kalintchenko, A. Maksimchuk, S. P. D. Mangles, T. Matsuoka, S. R. Nagel, C. A. J. Palmer, J. Schreiber, K. T. Phuoc, A. G. R. Thomas, V. Yanovsky, L. O. Silva, K. Krushelnick, and Z. Najmudin, Nat. Phys. 6, 980 (2010).

14. S. Y. Chen, A. Maksimchuk, and D. Umstadter, Nature 396, 653 (1998).

15. T. Tajima and J. M. Dawson, Phys. Rev. Lett. 43, 267 (1979).

16. S. L. Dexheimer, (Ed.) Terahertz Spectroscopy (CRC Press, Rochester, New York, USA, 2007), p. 41.

17. K. Ravi, W. R. Huang, S. Carbajo, E. A. Nanni, D. N. Schimpf, E. P. Ippen, and F. X. Kärtner, Opt. Express 23, 5253 (2015).

18. K. Ravi, D. N. Schimpf, and F. X. Kärtner, Opt. Express 24, 25582 (2016).

19. J. A. Fülöp, Z. Ollmann, Cs. Lombosi, C. Skrobol, S. Klingebiel, L. Pálfalvi, F. Krausz, S. Karsch, and J. Hebling, Opt. Express 22, 20155 (2014).

20. F. Ahr, S. W. Jolly, N. H. Matlis, S. Carbajo, T. Kroh, K. Ravi, D. N. Schimpf, J. Schulte, H. Ishizuki, T. Taira, A. R. Maier, and F. X. Kärtner, Opt. Lett. 42, 2118 (2017).

21. S. Carbajo, J. Schulte, X. Wu, K. Ravi, D. N. Schimpf, and F. X. Kärtner, Opt. Lett. 40, 5762 (2015).

22. X. Wu, A.-L. Calendron, K. Ravi, C. Zhou, M. Hemmer, F. Reichert, D. Zhang, H. Cankaya, L. E. Zapata, N. H. Matlis, and F. X. Kärtner, Opt. Express 24, 21059 (2016).

23. L. J. Wong, A. Fallahi, and F. X. Kärtner, Opt. Express 21, 9792 (2013).

24. A. Fallahi, A. Yahaghi, and F. Kärtner, "MITHRA 1.0: A fullwave simulation tool for free electron lasers," arXiv:1612.033 10 (2016).

25. C. Kupitz, S. Basu, I. Grotjohann, R. Fromme, N. A. Zatsepin, K. N. Rendek, M. S. Hunter, R. L. Shoeman, T. A. White, D. Wang, D. James, J.-H. Yang, D. E. Cobb, B. Reeder, R. G. Sierra, H. Liu, A. Barty, A. L. Aquila, D. Deponte, R. A. Kirian, S. Bari, J. J. Bergkamp, K. R. Beyerlein, M. J. Bogan, C. Caleman, T.-C. Chao, C. E. Conrad, K. M. Davis, H. Fleckenstein, L. Galli, S. P. Hau-Riege, S. Kassemeyer, H. Laksmono, M. Liang, L. Lomb, S. Marchesini, A. V. Martin, M. Messerschmidt, D. Milathianaki, K. Nass, A. Ros, S. RoyChowdhury, K. Schmidt, M. Seibert, J. Steinbrener, F. Stellato, L. Yan, C. Yoon, T. A. Moore, A. L. Moore, Y. Pushkar, G. J. Williams, S. Boutet, R. B. Doak, U. Weierstall, M. Frank, H. N. Chapman, J. C. H. Spence, and P. Fromme, Nature 513, 261 (2014).
26. L. Wang, A. Fallahi, K. Ravi, and F. X. Kärtner, "Multi-mJ terahertz generation in periodically poled lithium-niobate by pulse recycling," submitted to CLEO (2018).

27. I. Hagop and G. D Goodno, High-power Laser Handbook, 1st ed. (McGraw-Hill, New York, USA, 2011).

28. L. E. Zapata, D. J. Ripin, and T. Y. Fan, Opt. Lett. 35, 1854 (2010).

29. B. A. Reagan, K. A. Wernsing, A. H. Curtis, F. J. Furch, B. M. Luther, D. Patel, C. S. Menoni, and J. J. Rocca, Opt. Lett. 37, 3624 (2012).

30. C. Baumgarten, M. Pedicone, H. Bravo, H. Wang, L. Yin, C. S. Menoni, J. J. Rocca, and B. A. Reagan, Opt. Lett. 41, 3339 (2016).

31. M. Hemmer, L. Zapata, F. Reichert, K. Zapata, A. Calendron, K. Hong, H. Cankaya, and F. Kaertner, in Advanced Solid State Lasers, OSA Technical Digest (online) (Optical Society of America, Washington DC, USA, 2015), paper AW3A.8.

32. L. E. Zapata, H. Lin, A.-L. Calendron, H. Cankaya, W. R. Huang, E. Granados, K.-H. Hong, and F. X. Kärtner, Opt. Lett. 40, 2610 (2015).

33. M. Hemmer, L. Zapata, Y. Hua, and F. X. Kaertner, in Lasers Congress 2016 (ASSL, LSC, LAC), OSA Technical Digest (online) (Optical Society of America, 2016), paper ATh4A.3.

34. M. Hemmer, unpublished.

35. T. Metzger, A. Schwarz, C. Y. Teisset, D. Sutter, A. Killi, R. Kienberger, and F. Krausz, Opt. Lett. 34, 2123 (2009).

36. M. Larionov and J. Neuhaus, in Advanced Solid State Lasers (ASSL) (2014), paper ATh2A.51.

37. P. Russbueldt, T. Mans, J. Weitenberg, H. D. Hoffmann, and R. Poprawe, Opt. Lett. 35, 4169 (2010).

38. B. E. Schmidt, A. Hage, T. Mans, F. Légaré, and H. J. Wörner, Opt. Express 25, 17549 (2017).

39. M. Strotkamp, F. Elsen, J. Löhring, M. Traub, and D. Hoffmann, Appl. Opt. 56, 2886 (2017).

40. R. Jung, J. Tuemmler, and I. Will, Opt. Express 24, 883 (2016).

41. T. Nubbemeyer, M. Kaumanns, M. Ueffing, M. Gorjan, A. Alismail, H. Fattahi, J. Brons, O. Pronin, H. G. Barros, Z. Major, T. Metzger, D. Sutter, and F. Krausz, Opt. Lett. 42, 1381 (2017).

42. S. Preußler, N. Wenzel, R.-P. Braun, N. Owschimikow, C. Vogel, A. Deninger, A. Zadok, U. Woggon, and T. Schneider, Opt. Express 21, 23950 (2013).

43. J. Zhang, J. Brons, M. Seidel, V. Pervak, V. Kalashnikov, Z. Wei, A. Apolonski, F. Krausz, and O. Pronin, in European Conference on Lasers and Electro-Optics (2015), paper PD_A_1.

44. C. Wandt, S. Klingebiel, M. Schultze, S. Prinz, C. Y. Teisset, S. Stark, C. Grebing, M. Häfner, R. Bessing, T. Herzig, A. Budnicki, D. Sutter, K. Michel, T. Nubbemeyer, F. Krausz, and T. Metzger, in Conference on Lasers and Electro-Optics, OSA Technical Digest (online) (Optical Society of America, 2017), paper STh1L.1.

45. R. Jung, J. Tümmler, and I. Will, Opt. Express 24, 883 (2016).

46. T. Y. Fan, IEEE JSTQE 13, 448 (2007).

47. D. N. Schimpf, C. Ruchert, D. Nodop, J. Limpert, A. Tünnermann, and F. Salin, Opt. Express 16, 17637 (2008).

48. W. Liu, D. N. Schimpf, T. Eidam, J. Limpert, A. Tünnermann, F. X. Kärtner, and G. Chang, Opt. Lett. 40, 151 (2015).

49. H.-W. Chen, J. K. Lim, S.-W. Huang, D. N. Schimpf, F. X. Kärtner, and G. Chang, Opt. Express 20, 28672 (2012).

50. A.-L. Calendron, H. Cankaya, and F. X. Kärtner, Opt. Express 22, 24752 (2014).

51. C. Rouyer, E. Mazataud, I. Allais, A. Pierre, S. Seznec, C. Sauteret, G. Mourou, and A. Migus, Opt. Lett. 18, 214 (1993).

52. K. Beil, S. Fredrich-Thornton, F. Tellkamp, R. Peters, C. Kränkel, K. Petermann, and G. Huber, Opt. Express 18, 20712 (2010). 
53. E. B. Treacy, IEEE JQE 5, 454 (1969).

54. A.-L. Calendron, "Towards an ytterbium based optical waveform synthesizer," Dissertation (Universität Hamburg, 2015).

55. E. Esarey, S. K. Ride, and P. Sprangle, Phys. Rev. E 48, 3003 (1993).

56. P. Reckenthaeler, M. Centurion, V. S. Yakovlev, M. Lezius, and F. Krausz, Phys. Rev. A 77, 042902 (2008).

57. S. H. Brewer and S. Franzen, Chem. Phys. 299, 285 (2004).

58. G. H. Kassier, K. Haupt, N. Erasmus, E. G. Rohwer, H. M. von Bergmann, H. Schwoerer, S. M. M. Coelho, and F. D. Auret, Rev. Sci. Instrum. 81, 105103 (2010).

59. T. R. Schibli, J. Kim, O. Kuzucu, J. T. Gopinath, S. N. Tandon, G. S. Petrich, L. A. Kolodziejski, J. G. Fujimoto, E. P. Ippen, and F. X. Kaertner, Opt. Lett. 28, 947 (2003).

60. J. Kim, J. Chen, Z. Zhang, F. N. C. Wong, F. X. Kärtner, F. Loehl, and H. Schlarb, Opt. Lett. 32, 1044 (2007).
61. D. Zhang, A. Fallahi, X. Wu, M. Fakhari, H. Cankaya, A.L. Calendron, C. Zhou, W. R. Huang, D. Haynes, F. Lemery, F. Ahr, W. Qiao, N. H. Matlis, and F. X. Kärtner, in CLEO Europe (2017), oral presentation CC-1.4.

62. S. Zahedpour, J. K. Wahlstrand, and H. M. Milchberg, Opt. Lett. 40, 5794 (2015).

63. A.-L. Calendron, H. Cankaya, G. Cirmi, and F. X. Kärtner, Opt. Express 23, 13866 (2015).

64. D. E. Miller, L. E. Zapata, D. J. Ripin, and T. Y. Fan, Opt. Lett. 37, 2700 (2012).

65. P. Kroetz, A. Ruehl, A.-L. Calendron, G. Chatterjee, H. Cankaya, K. Murari, F. X. Kärtner, I. Hartl, and R. J. D. Miller, Appl. Phys. B 123, 126 (2017).

66. L. Indra, F. Batysta, P. Hř́bek, J. Novák, Z. Hubka, J. T. Green, R. Antipenkov, R. Boge, J. A. Naylon, P. Bakule, and B. Rus, Opt. Lett. 42, 843 (2017).

67. J. Galinis, G. Tamošauskas, I. Gražulevičiūtė, E. Keblytė, V. Jukna, and A. Dubietis, Phys. Rev. A 92, 033857 (2015). 